This item was submitted to Loughborough's Research Repository by the author.

Items in Figshare are protected by copyright, with all rights reserved, unless otherwise indicated.

\title{
M-integral analysis for cracks in a viscoplastic material with extended finite element method
}

PLEASE CITE THE PUBLISHED VERSION

https://doi.org/10.1016/j.engfracmech.2018.05.042

\section{PUBLISHER}

Elsevier

VERSION

AM (Accepted Manuscript)

\section{PUBLISHER STATEMENT}

This work is made available according to the conditions of the Creative Commons Attribution-NonCommercialNoDerivatives 4.0 International (CC BY-NC-ND 4.0) licence. Full details of this licence are available at: https://creativecommons.org/licenses/by-nc-nd/4.0/

\section{LICENCE}

CC BY-NC-ND 4.0

\section{REPOSITORY RECORD}

Hou, Junling, Hong Zuo, Qun Li, Rong Jiang, and Liguo Zhao. 2019. "M-integral Analysis for Cracks in a Viscoplastic Material with Extended Finite Element Method". figshare. https://hdl.handle.net/2134/33391. 


\title{
$M$-integral analysis for cracks in a viscoplastic material with extended
}

\section{finite element method}

\author{
Junling Hou ${ }^{\mathrm{a}}$, Hong Zuo ${ }^{\mathrm{a} *}$, Qun $\mathrm{Li}^{\mathrm{a}}{ }^{\mathrm{a}}$, Rong Jiang ${ }^{\mathrm{b}}$ and Liguo Zhao \\ ${ }^{a}$ State Key Laboratory for Strength and Vibration of Mechanical Structures, Shaanxi Key \\ Laboratory of Environment and Control for Flight Vehicle, Shaanxi Engineering Research \\ Center of Nondestructive Testing and Structural Integrity Evaluation, School of Aerospace, \\ Xi'an Jiaotong University, Xi'an 710049, China \\ ${ }^{\mathrm{b}}$ College of Energy and Power Engineering, Nanjing University of Aeronautics and \\ Astronautics, Nanjing, 210016, China \\ ${ }^{\mathrm{c}}$ Wolfson School of Mechanical, Electrical and Manufacturing Engineering, Loughborough \\ University, Loughborough, LE11 3TU, UK
}

\begin{abstract}
The $M$-integral can be used to quantify complex damage in materials subjected to mechanical deformation. However, the effect of viscoplasticity on the damage level associated with the $M$-integral has not been studied yet. In this paper, the variation of the $M$ integral associated with viscoplastic deformation was investigated numerically using a userdefined material subroutine. Effects of creep deformation and loading rate on the $M$-integral were also evaluated. In particular, the association of crack growth with the evolution of the $M$-integral was captured by the extended finite element method for different crack configurations. It was found that viscoplastic deformation has a great effect on the damage evolution of viscoplastic materials characterized by the $M$-integral. Crack growth leads to an increase of the $M$-integral, indicating progressive damage of the materials. Concerning the secondary cracks formed around a major crack, the results show that the $M$-integral is highly dependent on the numbers and locations of those secondary cracks. Shielding effect is mostly evident for microcracks with centres located just behind or vertically in line with the major
\end{abstract}


crack tip. With the increasing number of microcracks, the shielding effect tends to decrease as reflected by the increasing $M$-integral values.

Keywords: The $M$-integral; Viscoplastic material model; Multiple cracks; Extended finite element method; Crack growth; Shielding effect.

\section{Introduction}

Mechanical performance of materials is largely limited by the existence of defects such as cracks, voids, inclusions and dislocations [1-6]. For viscoplastic or time-dependent inelastic materials, it has been shown that viscoplastic deformation plays an important role in determination of stress/strain fields near a crack tip. Advanced constitutive models were generally adopted to describe the mechanical behaviour, study crack tip deformation and predict crack growth in viscoplastic materials. For instance, Lee et al. [7] introduced a viscoplastic model that considered the martensitic phase transformation of austenitic stainless steel to predict the material fracture under arbitrary loads. Through a user-defined subroutine, the results of the finite element simulations showed that the characteristics of crack propagation in an austenitic stainless-steel plate could be predicted effectively by their viscoplastic model. Chen and Liu [8] used a viscoplastic cohesive model to analyse the mechanical state and temperature increase in near-tip zone during steady crack propagation. Their model predictions showed a good consistency with the experimental results, in terms of temperature increase in the cohesive zone. In addition, the unified Chaboche viscoplastic model [9] was frequently used to simulate the time-dependent plastic deformation of nickel based superalloys, with model parameters optimised against monotonic, cyclic and creep experimental data. The model was also applied to study the stress-strain fields near a crack tip, revealing the near-tip strain accumulation feature under fatigue. The accumulated strain was subsequently used as a criterion to predict time-dependent crack growth rate in nickel alloys 
under fatigue loading, which was in good agreement with experimental results [10]. The model was also used in Farukh et al. [11] to predict the full process of fatigue crack growth in a nickel based superalloy, with the assistance of extended finite element method (XFEM). Again, model predictions compared very well with experimental results.

In order to describe the damage evolution in engineering materials, several methods have been proposed [12-16]. Among these, the energy parameters are of great interest due to their advantage in describing the complex fracture state of materials. By combining the XFEM and interaction energy integral, stress intensity factors can be obtained during the whole process of crack propagation [17-19]. For instance, Dolbow and Gosz [17] proposed an interaction energy integral method for the computation of stress intensity factors of a mixed-mode crack in functionally graded materials. Yu et al. [18] developed an interaction integral method to calculate stress intensity factors for an interface crack, interacting with a near-tip inclusion, in bi-materials under impact loading condition. Yu and Kitamura [19] presented a new domainindependent interaction integral (DII-integral) based on the assumption of a zero-mean stress field in order to consider the domain-dependence induced by the interface with thermomechanical properties. In addition, the well-known energy-conservation contour integrals, such as the $J_{k^{-}}$and $L$-integrals $[20,21]$ derived from Noether's theorem, also received a lot of attentions during the past years. For instance, the $J_{k}$-integrals $(k=1,2)$ have been widely used as energy fracture parameters to study the near-tip stress-strain state for crack problems [21]. However, the interaction methods mentioned above are limited to single crack problems, and not suitable for characterizing the energy state of multi-cracked bodies as it is unrealistic to track the behaviour of each individual crack tip. On the contrary, $M$-integral has a particular advantage in quantifying damage evolution for multiple cracks as it is calculated along a contour enclosing all cracks instead of a single crack tip. 
For the applications of the engineering safety prediction, the $M$-integral has attracted more attention in recent years owe to its practical importance in predicting the crack stability and growth. Particularly, it can be used to assess damage for multiple cracks in linear elastic. For instance, Chen [22] discussed the problem of multiple microcracks in an infinite 2D brittle solid, and concluded that the $M$-integral calculated along a closed contour surrounding all cracks can be divided into two parts, with the first part related to the stress intensity factors (SIFs) and the second part controlled by the two components of the $J_{k}$ integral. Moreover, based on the concept of the $M$-integral, a fracture parameter based on the critical value of the $M$-integral $(M c)$ was proposed by Chang and Lin [23], which was shown useful for evaluation of the surface energy associated with the creation of multiple curved cracks in $2 \mathrm{D}$ rubbery solid under large deformation. Based on the theory of configurational force, Yu and Li [24] physically interpreted the $M$-integral as self-similar expansion of a specific defect and subsequently proposed a new failure theory to investigate the evolution of multi-defects in a body. The application of $M$-integral for three dimensional models has also been confirmed in recent research efforts. In particular, based on the two-dimensional framework of $M$-integral, Chang et al. [25] reformulated a new invariant integral named as $M_{c}$-integral to evaluate the material fracture and structural integrity in three-dimensional (3D) elastic solids. And the finite element solutions of the 3D $M_{c}$-integral showed good accuracy and effectiveness. In summary, the $M$-integral can serve as an energy fracture parameter, but its application for the investigation of multi-cracked problems is still limited, especially the connection between the $M$-integral and crack growth.

A number of computational methods have been developed to study crack growth problems, for example cohesive element [26] the XFEM [27,28] and meshless methods [29]. Among these methods, it is known that crack growth can be simulated effectively by the XFEM without remeshing. In recent years, the XFEM method has been widely applied to a variety of 
crack problems, such as crack branching problem, fatigue crack growth and the evolution of multiple cracks. For instance, Daux et al. [30] verified the effectiveness of XFEM in assessing the SIFs for different models involving branched and intersecting cracks. Budyn et al. [31] used XFEM for modelling the growth behaviour of multiple cracks in a linear elastic media. They considered randomly distributed cracks in both homogeneous and nonhomogeneous materials, and the method was shown to be efficient and accurate for prediction of crack coalescence and percolation. Using the XFEM, Singh et al. [32] evaluated the fatigue life of homogeneous plate with multiple defects such as holes, cracks and inclusions. The SIFs for model with different discontinuities were calculated under cyclic loading, and the results obtained by XFEM agreed well with the experimental and remeshingbased FE solutions. Zhuang et al. [33] proposed an enhanced XFEM to investigate the crack branching phenomenon widely observed in engineering application. They developed two kinds of branching elements, named as "element crossed by two separated cracks" and "element embedded by a junction", to simulate the growth of a branched mode I crack.

Recently, some efforts have been made to study fracture toughness for viscoplastic materials using XFEM technique [34-36], and confirmed the validity of XFEM for viscoplasticity. For instance, Kastner et al. [34] investigated the effective viscoplastic behaviour of a unidirectional (UD) composite, based on XFEM simulation of representative volume element (RVE) and numerical homogenization technique. A good consistency was obtained between numerical and experimental results. Bergara et al. [36] studied the complex crack problem in a rectangular section of a viscoplastic beam and used XFEM as a new avenue to calculate stress intensity factors, which were in good agreement with experimental results.

However, so far there are no published papers in assessing damage evolution in viscoplastic materials using a combination of the $M$-integral (damage level) and XFEM (crack growth). By combining the $M$-integral and XFEM, it is expected to provide an improved 
understanding of the failure behaviour of these materials and support their engineering applications. This paper evaluated the damage of a viscoplastic material, containing single and multiple cracks, using the $M$-integral and XFEM. The same viscoplastic model and XFEM technique have been adopted by Farukh et al. [11] to model fatigue crack propagation under three-point bending, in good agreement with experimental results in terms of crack growth rate and path. However, there are no attempts made for $M$-integral analyses in either viscoplastic or viscoelastic materials. There are no results available for comparison in the open literatures.

The paper is constructed as follows. A brief description of the $M$-integral and XFEM is given in Section 2. Section 3 explains the formulation of a viscoplastic model used in this paper. Numerical simulations are presented in Section 4 to study the damage evolution based on the analysis of the $M$-integral. Specifically, the effects of plasticity, creep and loading rate on damage evolution were studied by the invariant parameter $M$-integral for the single crack problem. The analyses were then extended to the multiple-crack problem. The main conclusions are summarized in Section 5.

\section{The $M$-integral and extended finite element method}

\subsection{Derivation of the $M$-integral}

Based on material configurational force theory $[37,38]$, the $M$-integral can be physically interpreted as the potential energy change during the self-similar expansion of the defects. The invariant $M$-integral has been widely applied to predict the stability of multiple defects $[39,40]$. Since the $M$-integral is inherently related to the change of the total potential energy for a damaged material without concerning the specific damage information of every defect, it is an effective method to evaluate the damage evolution of materials containing complex defects. For instance, Chen [39] presented a description of the $M$-integral for an infinite brittle solid containing multiple micro-cracks, which was associated with the Eshelby's 
energy momentum tensor and the corresponding invariant integrals such as $J_{k}$-integral and $L$ integral. Based on Chen's work [39], $M$-integral has been introduced as a damage parameter for evaluating the degradation of material and structural integrity induced by the evolution of multi-defects. For instance, Li et al. [40] successfully used $M$-integral to describe fracture of elastic-plastic material with multiple defects (e.g. voids) subjected to biaxial loading conditions. The feasibility of $M$-integral in evaluating multi-defect problems has been verified for different materials including piezoelectric, general anisotropic and elastic-plastic materials. In this study, we assessed $M$-integral for a viscoplastic material containing cracks. The results confirmed that $M$-integral is also an effective parameter in quantifying damage related to crack growth in viscoplastic materials.

For a multi-defect system as depicted in Fig.1, the $M$-integral is shown to be associated with the material configurational force. Firstly, it is well known that the Lagrangian density $L$ is a function related to coordinates $x_{i}(i=1,2)$, displacements $u_{k}$ and total strain tensor $\varepsilon_{k j}$. Therefore, in the absence of inertia terms and body force, the Lagrangian function $L$ can be identified as the negative strain energy density of the system,

$$
L=L\left(x_{i}, u_{k, j}\left(x_{i}\right)\right)=-W\left(x_{i}, u_{k, j}\left(x_{i}\right)\right)
$$

where $u_{k}$ represents the components of displacement, and the subscript prima "," refers to the differentiation of a variable with respect to the coordinate.

The $M$-integral can be obtained by taking the divergence of the Lagrangian function as follows,

$$
\nabla \square(L \mathbf{x})=\left(L x_{i}\right)_{, i}=-m W-\left(\frac{\partial W}{\partial x_{i}}\right)_{\operatorname{expl}} x_{i}-\sigma_{k j} u_{k, j i} x_{i}
$$

where $\left(\partial W / \partial x_{i}\right)_{\text {expl }}$ denotes the explicit dependence of $W$ on $x_{i}$. The parameter $m$ represents $x_{i, i}$ and is identical to 2 for two dimensions. $\sigma_{k j}$ refers to the Cauchy stress tensor. Considering the equilibrium condition, 


$$
\sigma_{k j, j}=0
$$

The Eq. (2) can be rewritten as

$$
\left[W x_{i} \delta_{i j}-\sigma_{j k} u_{k, i} x_{i}\right]_{, j}=m W-\sigma_{j k} u_{k, j}+\left(\frac{\partial W}{\partial x_{i}}\right)_{\operatorname{expl}} x_{i}
$$

Consequently, the derived configurational force equilibrium equation is obtained as,

$$
M_{j, j}+\Lambda=0
$$

with

$$
\begin{gathered}
M_{j}=W x_{i} \delta_{i j}-\sigma_{j k} u_{k, i} x_{i}+\frac{2-m}{2} \sigma_{j k} u_{k}, \\
\Lambda=\left(\frac{\partial W}{\partial x_{i}}\right)_{\operatorname{expl}} x_{i}
\end{gathered}
$$

Here, the term $M_{j}$ is defined as the damage expansion configurational force of material, which is related to the change of the potential energy during the formation of defects in the initially undamaged system. As described in [38], it is similar to the definition of the Eshelby material forces. And the term $\Lambda$ is treated as the source of material damage, which is induced by the discontinuities due to the presence of defects in materials.

The $M$-integral is associated with the integration of the configurational force $M_{j}$ along a closed contour. The global $M$-integral over the contour $\Gamma$ enclosing all defects can be interpreted as the sum of local energy release due to the self-similar expansion for each specific defect. Considering a two-dimensional space, integrating the component $M_{j, j}$ over area $A$, as shown in Fig. 1, leads to

$$
M=\int_{A} M_{j, j} d A=\int_{A}\left(W x_{i} \delta_{i j}-\sigma_{j k} u_{k, i} x_{i}\right)_{, j} d A \quad(i, j, k=1,2)
$$

Using Green's theorem, Eq. (8) can be rewritten as,

$$
M=\int_{\Gamma}\left(W x_{i} n_{i}-T_{k} u_{k, i} x_{i}\right) d l \quad(i, j, k=1,2)
$$


where $\Gamma$ is a counter-clockwise closed contour around the whole crack, $W$ is the strain energy density of the material, $T_{k}=\sigma_{k j} n_{j}$ is the traction vector, $n_{i}$ is the outward unit vector normal to $\Gamma, l$ is the arc length, $\boldsymbol{x}$ is the position vector of the integration point.

\subsection{Computation of the $M$-integral}

In the present study, numerical computations are performed to obtain the value of the $M$ integral for multi-crack problem in viscoplastic materials. Firstly, we obtain the all quantities of variables in Eq. (9) from the finite element method ABAQUS and the viscoplastic model presented in Section 3. Here, the variables refer to stress, strain and strain energy at Gauss integration points, and displacement at nodal points. And then, the values of $M$-integral for defined paths could be obtained by the numerical procedure of integration.

The $M$-integral is a linear integral and can be rewritten as:

$$
M=\int_{\Gamma}\left(W x_{i} n_{i}-T_{k} u_{k, i} x_{i}\right) d l=\int_{\Gamma} M_{i} n_{i} d \Gamma
$$

To calculate the $M$-integral numerically, a domain integral method was introduced. Basically, a closed contour integral is replaced with a domain integral performed over the area or volume enclosed by the contour. The method has been widely used for calculation of fracture parameters, such as 3D energy release rate, interaction I-integral and $J$ integral [41-43].

To start with, a weight function $q$ is introduced firstly. Here, $q$ denotes an arbitrary function which could go through the area $A$ smoothly, as shown in Fig. 2.

The identity relation is given as:

$$
\left(M_{i} q\right)_{, i}=M_{i, i} q+M_{i} q_{, i}
$$

Integrating the Eq. (11) over an area $A$ and applying the divergence theorem, we could obtain

$$
\int_{\Gamma} M_{i} q n_{i} d \Gamma=-\int_{A}\left(M_{i, i} q+M_{i} q_{, i}\right) d A
$$

Here, the integral area $A$ is defined as the shadow region between two closed contours $\Gamma$ and $\Gamma_{0}$ (see Fig. 2). The value of $q$-function is set as 0 and 1 on outer $(\Gamma)$ and inner $\left(\Gamma_{0}\right)$ contours, 
respectively. It has been demonstrated that the selection of $q$ values on the inner and outer boundaries $\left(\Gamma_{0}\right.$ and $\left.\Gamma\right)$ are not sensitive to the calculation of the $M$-integral, as it is just a transition function [41-43].

Combining Eqs. (10) and (12), and introducing $M_{i, i}=0$ in the zone $A$ due to free of singularity, we have

$$
M=-\int_{A}\left(M_{i, i} q+M_{i} q_{, i}\right) d A=\int_{A}\left(-W x_{i}+\sigma_{k i} u_{k, j} x_{j}\right) \frac{\partial q}{\partial x_{i}} d A
$$

The function $q$ could be interpolated over the whole element with the aid of shape functions,

$$
q=N_{1} q_{1}^{e}+N_{2} q_{2}^{e}+N_{3} q_{3}^{e}+N_{4} q_{4}^{e}=\mathbf{N q}^{e}=\sum_{i=1}^{4} N_{i}(r, s) q_{i}^{e}
$$

where $N_{i}$ is the shape function related with coordinates $(x, y)$ of the element, and $(r, s)$ represents the position of Gauss point. Thus, the $M$-integral in a single element could be obtained by

$$
M^{e}=\int_{-1}^{1} \int_{-1}^{1} I(r, s) d r d s
$$

with

$$
I(r, s)=\left[\begin{array}{l}
\left(\sigma_{x x} \frac{\partial u}{\partial x} x+\sigma_{x x} \frac{\partial u}{\partial y} y+\tau_{x y} \frac{\partial v}{\partial x} x+\tau_{x y} \frac{\partial v}{\partial y} y-W x\right) \frac{\partial q}{\partial x} \\
+\left(\tau_{x y} \frac{\partial u}{\partial x} x+\tau_{x y} \frac{\partial u}{\partial y} y+\sigma_{y y} \frac{\partial v}{\partial x} x+\sigma_{y y} \frac{\partial v}{\partial y} y-W y\right) \frac{\partial q}{\partial y}
\end{array}\right] \operatorname{det}\left(\mathbf{J}^{\mathbf{e}}\right)
$$

where $\operatorname{det}\left(\mathbf{J}^{\mathrm{e}}\right)$ denotes the determinant of Jacobi matrix for the corresponding element. Using Gauss integral method, the Eq. (15) could be approximately obtained by the sum of the values of $I(r, s)$ on Gauss points:

$$
M^{e} \approx I\left(r_{1}, s_{1}\right)+I\left(r_{2}, s_{2}\right)+I\left(r_{3}, s_{3}\right)+I\left(r_{4}, s_{4}\right)
$$


Here, four Gauss points were used in the integration for the entire domain in this study. In addition, as shown in Fig. 2, the integral domain $A$ is an annular area which does not involve any cracked elements. Basically, $M$-integral computation was performed far away from the cracked region. Numerical calculations show that the results of $M$-integral are not sensitive with the crack tip fields. Therefore, no special technique is required for the crack tip. By repeating the above procedure, the value of the $M$-integral corresponding to the chosen integral contour could be obtained by the sum of $M^{e}$ within the enclosed path. Therefore, in order to avert the unexpected path-dependent issues, the present study assumes that the nonlinear deformation is localized inside a damaged viscoplastic zone located at the centre of the plate while the outer region is assumed to be linear elasticity with infinitesimal elastic modulus (Fig. 3). Here, damage zone refers to a region which contains all cracks and plastic deformation. It should be mentioned that damage zone adopted in the computation of numerical examples should be sufficiently large to ensure the path-independency of $M$ integral $[24,40,44,45]$. However, the actual size of the damage zone does not affect numerical calculation of $M$-integral which is performed over area " $A$ " free of defects (see Fig. 2).

\subsection{Descriptions of XFEM}

The XFEM was developed by Belytschko and co-workers in 1999 [46]. It is an extension of the standard finite element method based on the concept of partition of unity, and is useful for the approximation of solutions with pronounced non-smooth characteristics in small parts of the computational domain, for example, near discontinuities and singularities. In the XFEM, a crack is modelled in an element by enriching degrees of freedom with special displacement functions. The displacement approximation for an arbitrary Gauss point in one element in the XFEM takes the following form: 


$$
\mathbf{u}=\sum_{i=1}^{n} N_{i}(\mathbf{x})\left[\mathbf{u}_{i}+H(\mathbf{x}) \mathbf{a}_{i}+\sum_{\alpha=1}^{4} F_{\alpha}(\mathbf{x}) \mathbf{b}_{i \alpha}\right]
$$

where $\mathbf{u}$ represents the total displacement vector, $n$ is the number of nodes in the mesh, $N_{i}(\mathbf{x})$ is the shape function of node $i, \mathbf{u}_{i}$ are the nodal displacement vectors, and $\mathbf{a}_{i}$ and $\mathbf{b}_{i \alpha}$ are the nodal enriched degree of freedom vector associated with the Heaviside step function $H(\mathbf{x})$ and the asymptotic crack-tip functions $F_{\alpha}(\mathbf{x})$, respectively. The $H(\mathbf{x})$ is the jump function, defined as

$$
H(x)=\left\{\begin{array}{cc}
1 & x \geq 0 \\
-1 & x<0
\end{array}\right.
$$

The crack-tip functions $F_{\alpha}(\mathbf{x})$ provide improved accuracy and are required if the crack-tip terminates inside an element. These functions are

$$
\left[F_{\alpha}(r, \theta), \alpha=1-4\right]=\left[\sqrt{r} \sin \frac{\theta}{2}, \sqrt{r} \cos \frac{\theta}{2}, \sqrt{r} \sin \frac{\theta}{2} \sin \theta, \sqrt{r} \cos \frac{\theta}{2} \sin \theta\right],
$$

where $r, \theta$ are local polar coordinates defined at the crack tip as shown in Fig. 4, in which the crack tip is the pole and the polar axis is along the crack propagation direction. The enrichment functions are suitable for viscoplastic materials. These enrichment functions have already successfully used to deal with the crack problems in viscoplastic materials [47-49]. Additionally, the enrichment functions are also suitable for the case of micro-crack and interacting cracks.

XFEM defines the elements containing at least one enriched node as enriched elements. Nodes of the cracked-element surrounded by yellow contour in Fig. 4 have shape functions that multiply $H(\mathbf{x})$. Nodes of the tip-element represented by the blue square are enriched in the two Cartesian directions with $F_{\alpha}(\mathbf{x})$.

\section{Viscoplastic model}

\subsection{Model description}


The viscoplastic model employed in this study is one of the classical constitutive relationships based on the consideration of yielding. The model was originally developed by Chaboche [9], who used both kinematic ( $\boldsymbol{\alpha})$ and isotropic $(\mathrm{R})$ hardening variables to describe the full stages of cyclic stress-strain response. The model is proved capable of describing time-dependent cyclic plastic deformation for a series of metallic materials such as stainless steels and nickel alloys considered in this work.

For small strain regime, the strain rate tensor is additively decomposed into two parts, i.e., the elastic $\left(\dot{\boldsymbol{\varepsilon}}_{e}\right)$ and the inelastic $\left(\dot{\boldsymbol{\varepsilon}}_{p}\right)$ parts:

$$
\dot{\boldsymbol{\varepsilon}}=\dot{\boldsymbol{\varepsilon}}_{e}+\dot{\boldsymbol{\varepsilon}}_{p}
$$

The elastic part of the strain ( $\left.\dot{\boldsymbol{\varepsilon}}_{e}\right)$ follows the Hook's law,

$$
\dot{\boldsymbol{\varepsilon}}_{e}=\frac{1+v}{E} \dot{\boldsymbol{\sigma}}-\frac{v}{E}(t r \dot{\boldsymbol{\sigma}}) \mathbf{I}
$$

where $\boldsymbol{\sigma}$ and $\mathbf{I}$ are the stress tensor and the unit tensor of order two, $t r$ the trace, and $E$ and $v$ are the Young's modulus and the Poisson's ratio, respectively.

The inelastic part of the strain $\left(\boldsymbol{\varepsilon}_{p}\right)$ consists of both plastic and creep components, and its rate can be expressed as [9]:

$$
\dot{\boldsymbol{\varepsilon}}_{p}=\left\langle\frac{f}{Z}\right\rangle^{m} \frac{\partial f}{\partial \boldsymbol{\sigma}}
$$

where $m$ and $Z$ are viscous constants, $f$ is the yield function, and the bracket is defined as:

$$
\langle x\rangle=\left\{\begin{array}{l}
x, x \geq 0 \\
0, x<0 .
\end{array}\right.
$$

According to the yield criterion of von Mises, the yielding function is given by

$$
f(\sigma, \alpha, R, k)=J(\sigma-\alpha)-R-k \leq 0
$$

where $\boldsymbol{\alpha}$ and $R$ are the hardening variables, $k$ is the initial radius of the yield surface, and $J$ is given by: 


$$
J(\boldsymbol{\sigma}-\boldsymbol{\alpha})=\sqrt{\frac{3}{2}\left(\boldsymbol{\sigma}^{\prime}-\boldsymbol{\alpha}^{\prime}\right):\left(\boldsymbol{\sigma}^{\prime}-\boldsymbol{\alpha}^{\prime}\right)}
$$

In Eq. (26), $\boldsymbol{\alpha}^{\prime}$ and $\boldsymbol{\sigma}^{\prime}$ refer to the deviators of $\boldsymbol{\alpha}$ and $\boldsymbol{\sigma}$, and : denotes the product of tensors.

Plastic deformation occurs only when $f=0$ and $\frac{\partial f}{\partial \boldsymbol{\sigma}}: \dot{\boldsymbol{\sigma}}>0$. The kinematic $(\boldsymbol{\alpha})$ and isotropic $(R)$ hardening variables are described by [9]:

$$
\left\{\begin{array}{l}
\dot{\boldsymbol{\alpha}}=\dot{\boldsymbol{\alpha}}_{1}+\dot{\boldsymbol{\alpha}}_{2} \\
\dot{\boldsymbol{\alpha}}_{1}=C_{1}\left(a_{1} \dot{\boldsymbol{\varepsilon}}_{\mathbf{p}}-\dot{\boldsymbol{\alpha}}_{1} \dot{p}\right) \quad \text { and } \quad \dot{R}=b(Q-R) \dot{p} \\
\dot{\boldsymbol{\alpha}}_{2}=C_{2}\left(a_{2} \dot{\boldsymbol{\varepsilon}}_{\mathbf{p}}-\dot{\boldsymbol{\alpha}}_{2} \dot{p}\right)
\end{array}\right.
$$

where $C_{1}, a_{1}, C_{2}, a_{2}, b$ and $Q$ are material constants which decide the stress-strain loops during cyclic loading, and the accumulated inelastic strain rate $\dot{p}$ is given by:

$$
\dot{p}=\left\langle\frac{f}{Z}\right\rangle^{n}=\sqrt{\frac{2}{3} \mathrm{~d} \dot{\boldsymbol{\varepsilon}}_{\mathbf{p}}: \mathrm{d} \dot{\boldsymbol{\varepsilon}}_{\mathbf{p}}}
$$

\subsection{Material and model parameters}

The material considered in this study is the nickel-based superalloy LSHR (Low Solvus High Refractory) which experiences viscoplastic deformation under the sustained loading and/or dwell-fatigue loading, especially at elevated temperatures. Nickel-based superalloys are widely used for blades and discs in gas turbine at high temperatures, since they were designed to provide superior combination of properties such as high temperature strength, exceptional fatigue and creep resistance, and good thermal performance. The turbine blades or discs are subjected to varying centrifugal and thermal stresses at high temperatures during service, which causes fatigue, creep and environmental damage. Therefore, understanding the crack initiation and growth in these alloys at high temperatures is crucial for ensuring the structural integrity of gas turbines.

The viscoplastic model parameters were optimized by Farukh et al. [11] against the uniaxial tensile test data of $\mathrm{LSHR}$ at $725^{\circ} \mathrm{C}$, with values given in Table 1 for completeness. Simulated 
strain-rate effects of four different strain rates $\left(0.001 / \mathrm{s}, 0.01 / \mathrm{s}, 0.1 / \mathrm{s}\right.$ and $\left.8.3 \times 10^{-5} / \mathrm{s}\right)$ are shown in Fig. 5 for monotonic tensile (maximum strain 1\%). A comparison of the simulated response and experimental data was also included for a strain rate of $8.3 \times 10^{-5} / \mathrm{s}$, which shows a good agreement between the two.

The above model has already been programmed into a user defined material subroutine (UMAT) and implemented into the finite element code ABAQUS [10]. The computational cost is between 10 and 40 minutes for most of examples simulated using single CPU i7 intel core (i7-6700 CPU@ 3.4GHz). Specifically, for the model containing one single crack in Section 4.1 and 4.2 (including the creep analysis), a job in ABAQUS could be completed within 40 minutes. The computational times in Section 4.3 are very similar to those in Section 4.1. The shielding analysis in Section 4.4 took about 10 to 20 minutes to finish, depending on the number of microcracks.

\section{Numerical results and discussions}

\subsection{Effect of viscoplasticity}

Firstly, we considered a plate with a central single crack, under plane strain condition and subjected to uniform tension $\sigma$ on its top side, as shown in Fig. 6a. The full dimension of the 2D plate is $45 \mathrm{~mm} \times 45 \mathrm{~mm}$ (length $\times$ width), while the dimension of the damage zone is 20 $\mathrm{mm} \times 20 \mathrm{~mm}$ (length $\times$ width). The centre crack has a length of $3 \mathrm{~mm}$. The finite element mesh is shown in Fig. 6b, consisting of four-node plane-strain elements with full integration. In this problem, the applied tensile stress $\sigma$ is $500 \mathrm{MPa}$ over a period of 100 seconds. The criterion applied in XFEM to control crack growth is the maximum principle stress criterion, with a critical value of $800 \mathrm{MPa}$ based on uniaxial tensile testing. And the angle of crack propagation is perpendicular to the direction of the maximum principal stress. For direct comparison, simulations were carried out for both elastic and viscoplastic material models. 
The elastic model has a Young's modulus of $178 \mathrm{GPa}$ and a Poisson's ratio of 0.285 . The viscoplastic model was described in Section 3.

As shown in Fig. 7, the line with black symbols represents the evolution of the $M$-integral in an elastic material, while the line with red symbols denotes the results for a viscoplastic material. In general, the $M$-integral for the two material models increased monotonically with the increasing load level, but with a difference. The values of the $M$-integral in viscoplastic model are higher than those in the pure elastic model. The $M$-integral denotes the degree of damage for the material with defects. Specifically, the plastic deformation enclosed by the a rectangle integral path, with a length of $25 \mathrm{~mm}$ and a width of $26.5 \mathrm{~mm}$ (shown in Fig. 3) made additional contributions to the value of the $M$-integral. Compared to the pure elastic model, there's no plastic strain. For instance, after a time point of $67 \mathrm{~s}$, the difference between viscoplastic model and pure elastic model became very distinct. At a time point of $100 \mathrm{~s}$, the value of the $M$-integral was $25.33 \mathrm{~J} / \mathrm{m}$ for pure elastic material model and increased to $29.12 \mathrm{~J} / \mathrm{m}$ for viscoplastic model, i.e., an increase of nearly $15 \%$.

The XFEM-based simulations enabled the modelling of crack growth, and the crack was seen to propagate steadily after a time point of 39 seconds. As shown in Fig. 8, the increase of the $M$-integral is directly linked with amount of crack growth for both material models. Longer crack resulted in higher value of the $M$-integral, which indicates the increased degree of damage. The results confirmed the effectiveness of the $M$-integral as a parameter in evaluating the damage of viscoplastic material during crack growth. The crack propagation predicted at the end of loading is shown in Fig.8 for two material models. Furthermore, the crack propagates mainly along the crack surface as the geometrical model is a standard Mode I crack problem, with an approximate growth of $1.2 \mathrm{~mm}$ for each crack tip at the end of loading. 
To verify the path-independence of $M$-integral for viscoplastic material, ten different rectangular paths were chosen to compute $M$-integral. Specifically, the size of the 10 rectangle paths varied between $25 \mathrm{~mm}$ and $26.5 \mathrm{~mm}$ for both length and width (see Fig. 3). The results are given in Table 2, and confirm that $M$-integral has a conservation nature (with a difference of less than $0.9 \%$ ) for a viscoplastic material.

\subsection{Time dependency of the $M$-integral in a viscoplastic material}

Numerical simulations were carried out to investigate the time dependency of the $M$-integral in viscoplastic materials. The geometry model used is the same as that in Section 4.1. Two loading cases were considered in the simulations. One is to investigate the influence of creep deformation, and the other one is to look at the effect of loading rate.

\subsubsection{Effect of creep deformation}

Creep deformation was simulated by subjecting the plate to a constant load over a sufficiently long period of times. As depicted in Fig. 9, there are two loading steps in the finite element analysis. Specifically, loading in step I was monotonic with a peak value of $500 \mathrm{MPa}$ while loading in step II was held at a constant level of $500 \mathrm{MPa}$. The corresponding step time was $50 \mathrm{~s}$ and two days, respectively. The evolution of the $M$-integral is presented in Fig. 9 for the two loading steps. See loading step I shown in Fig. 9, under monotonic loading, the value of the $M$-integral increases up to $28.30 \mathrm{~J} / \mathrm{m}$. During loading step II, the value of the $M$-integral increases slowly over time, e.g., from $31.47 \mathrm{~J} / \mathrm{m}$ to $33.95 \mathrm{~J} / \mathrm{m}$. The results indicate the accumulation of material damage over creep deformation. However, the increase is a slow process, indicating limited creep deformation of the material.

Distributions of von Mises stress for the entire domain were presented in Fig. 10 at different times of creep deformation. From $22.3 s$ to $50 s$ (Figs. 10a-10f), the model was under loading stage with increasing load level. However, the maximum stress at $28.2 \mathrm{~s}$ was found less than that at $22.3 \mathrm{~s}$. Combining with the variation of $M$-integral shown in Fig. 9, it is clear that the 
stress distribution was influenced by the crack growth in the material. Although $M$-integral showed an increasing trend from $0 s$ to $50 s$, the maximum value of von Mises stress may experience a sudden drop due to stress release associated with crack growth. During the creep deformation stage (Figs. $10 \mathrm{~g}$ and $10 \mathrm{~h}$ ), the von Mises stress decreased slightly. The distribution of displacement was also shown in Fig. 11 for the same time points. It can be seen that the magnitude of displacement increased gradually during the whole loading process as a response to crack growth.

For loading step I, the corresponding crack growth captured by XFEM is indicated a growth of $1.05 \mathrm{~mm}$ at each crack tip at the end of loading step. No crack growth was found for the loading step II (creep deformation). According to the evolution of the $M$-integral (loading step I \& II) against the amount of crack growth, the $M$-integral has a monotonic increase during loading step I. However, during creep deformation (load holding period), the $M$ integral only experienced a slight increase, reflecting the absence of crack growth.

\subsubsection{Effect of loading rate}

Three loading rates, $5 \mathrm{MPa} / \mathrm{s}, 50 \mathrm{MPa} / \mathrm{s}$ and $5000 \mathrm{MPa} / \mathrm{s}$, were considered to investigate the dependency of the $M$-integral on the loading rate. The peak stress is $500 \mathrm{MPa}$, and the corresponding step time for the employed loading rates is $100 s, 10 s$ and $0.1 s$ respectively. The computed $M$-integral against the normalised time is presented in Fig. 12 for the three loading rates, from which we can see that the degree of damage decreases with the increasing loading rate. This is because the higher loading rate would lead to a lower strain level in the material. For instance, the values of the $M$-integral at end of the loading step were calculated as $29 \mathrm{~J} / \mathrm{m}, 27.7 \mathrm{~J} / \mathrm{m}$ and $25.13 \mathrm{~J} / \mathrm{m}$ for three loading rates, $5 \mathrm{MPa} / \mathrm{s}, 50 \mathrm{MPa} / \mathrm{s}$ and $5000 \mathrm{MPa} / \mathrm{s}$, respectively. It should be noted that this phenomenon is valid for load-controlled loading condition. For displacement controlled loading condition, the degree of damage would increase with the increasing loading rate due to the higher level of stress. Also, as shown in 
Fig. 12, when the normalized time is more than 0.4 , it seems the $M$-integral corresponding to the loading rate of $5 \mathrm{MPa} / \mathrm{s}$ is higher than others. Obviously, the differences of $M$-integral for loading rate $=5 \mathrm{MPa} / \mathrm{s}$ could reach $4.7 \%$ and $15.4 \%$ compared to the values of $M$ corresponding to the loading rates of $50 \mathrm{MPa} / \mathrm{s}$ and $5000 \mathrm{MPa} / \mathrm{s}$. Therefore, the $M$-integral can directly indicate the influence of loading rate on the damage of viscoplastic material.

The variation of the $M$-integral with the crack growth is shown in Fig. 13 for three loading rates, from which two main conclusions emerges. Firstly, for the same amount of crack growth, lower loading rate induced higher damage energy (represented by the $M$-integral). For instance, when the crack had a propagation of $1.2 \mathrm{~mm}$, the value of $M$-integral is 29.00 $\mathrm{J} / \mathrm{m}$ for loading rate $5 \mathrm{MPa} / \mathrm{s}$ while $M=25.13 \mathrm{~J} / \mathrm{m}$ for loading rate $5000 \mathrm{MPa} / \mathrm{s}$. Secondly, the $M$-integral could be the same even through the amount of crack growth was different for three loading rates. For instance, for a loading rate of $5000 \mathrm{MPa} / \mathrm{s}$, the value of the $M$-integral was $18.94 \mathrm{~J} / \mathrm{m}$ when the original crack had a growth of $0.9 \mathrm{~mm}$. This value of the $M$-integral $(18.94 \mathrm{~J} / \mathrm{m})$ correspond to the crack growth of $0.78 \mathrm{~mm}$ and $0.82 \mathrm{~mm}$ for loading rates of 5 $\mathrm{MPa} / \mathrm{s}$ and $50 \mathrm{MPa} / \mathrm{s}$, respectively. Moreover, for the three loading rates, the corresponding crack growth, following the onset of crack propagation, is the same at the end of loading step, i.e., a growth of $1.2 \mathrm{~mm}$ at both crack tips at the end of loading time.

\subsection{The $M$-integral of two interacting cracks}

A plate containing two interacting cracks was considered, and the dimension of the plate and the loading condition are the same as that described in Section 4.1. Crack 1 has a length of 3 $\mathrm{mm}$ and is parallel to the $x$ axis. Crack 2 also has a length of $3 \mathrm{~mm}$, and its centre is positioned at the $x$-axis with a distance of $3 \mathrm{~mm}$ from the centre of Crack 1 . The angle $\alpha$ indicates the orientation of crack 2 with respect to $x$-axis, as shown in Fig. 14.

Fig. 15 shows the evolution of $M$-integral with varying angle $\alpha$. It is noted that the $M$-integral for $\alpha=30^{\circ}$ and $\alpha=150^{\circ}$ is almost the same, with a difference of less than $0.5 \%$ (Table 3; end 
of loading step). This is also the case for $\alpha=60^{\circ}$ and $\alpha=120^{\circ}$, confirming the symmetry of the model. Consequently, we only need to analyse the results between $0^{\circ}$ and $90^{\circ}$. From Table 3, the $M$-integral decreases when $\alpha$ increases from $0^{\circ}$ to $30^{\circ}$, with a sudden jump when $\alpha$ reaches $45^{\circ}$ and a steady decline afterwards.

Clearly, two collinear cracks induced the highest value of the $M$-integral (115.54 J/m). Profile of crack growth for two collinear cracks is given in Fig. 16. At the final state, both cracks propagated for $2.25 \mathrm{~mm}$ at the far tips. In addition, these two collinear cracks approached each other at the adjacent tips and became one crack with a growth of $1.5 \mathrm{~mm}$. From the crack growth behaviour captured by XFEM, it can be concluded that the two collinear cracks in material will coalescence, and finally form one long crack which would induce higher damage, i.e., significantly increased value of the $M$-integral.

\subsection{The $M$-integral and microcrack shielding effect}

Crack propagation is usually accompanied by the formation of microcracks in the plastic zone or process zone at the main crack tip. For instance, multiple slip band cracks which have certain inclined angles with respect to the main crack path are formed in the crack-tip plastic zone in a polycrystalline Ni-based superalloy at room temperature [50]. Also, secondary grain boundary cracks, formed around the main grain boundary crack and parallel to main crack plane (normal to loading direction), are observed in a polycrystalline Ni-based superalloy under the fatigue-oxidation conditions based on both 2D metallographic observation and 3D computed tomography examination $[51,52]$. These secondary cracks, either discontinuous or interlinked, strongly interact with the main crack. The interaction is usually associated with the redistribution and/or reduction of the near-tip stresses, leading to decelerated or accelerated crack propagation due to the crack tip shielding or amplification effects [53]. It is reported that microcracks have strong influences on the crack opening profile, especially when they are parallel to the main crack plane [54]. 
In this Section, the $M$-integral was used to investigate microcrack toughening effect, for which varied number and distribution of microcracks, near a main crack tip and parallel to the main crack plane, were considered. All simulations were assumed to be plane strain and under a uniaxial tensile stress of $500 \mathrm{MPa}$ (as previous sections). The model with a single main crack, as depicted in Fig. 6, was also simulated. The main crack has a length of $6 \mathrm{~mm}$ and the value of $M$-integral was computed as $138.36 \mathrm{~J} / \mathrm{m}$. This was used a reference state to evaluate the microcrack toughening effect in the following analyses.

Here, a plate containing a main crack and a vertical row of parallel microcracks was investigated. We assumed that all the added microcracks had the same length of $0.6 \mathrm{~mm}$, and separated by a space of $0.4 \mathrm{~mm}$ from each other. Micro-crack mentioned in this study refers to a much shorter length when compared to the length of the main crack. So, no special treatment or new parameter was required in the XFEM formulation. For instance, Loehnert and Belytschko [55] presented a multiscale method for simulations of crack propagation between macroscale and microscale, where the same XFEM technique was used to carry out simulations for both macrocarcks and microcracks. Guidault et al. [56] and Svenning et al. [57] also simulated the evolution of microcracks using XFEM method without any special treatment.

As depicted in Fig. 17, three sets of models were considered by varying the distance (denoted as $d$ ) between the centre line of the microcracks and the right tip of the main crack. Specifically, the centre line of the vertical row of microcracks was chosen to be located behind ( $d=0.3 \mathrm{~mm}$; Fig. 17a), in line with $(d=0 \mathrm{~mm}$; Fig. 17b) and ahead of $(d=0.3 \mathrm{~mm}$; Fig. 17c) the right tip of the main crack, respectively. The varied numbers of microcracks were $2,4,8,16$ and 32 .

As shown in Fig. 18, the lines with black, red and blue symbols represent the values of the $M$ integral against the number of added microcracks for the three models considered. The 
reference value of the $M$-integral (138.36 $\mathrm{J} / \mathrm{m}$ for a single main crack) was plotted as a dashed line to judge the shielding or amplification effects caused by the microcracks. Firstly, the relative position of microcracks to the main crack tip has a significant influence on the value of the $M$-integral. This indicates that the microcrack-toughening effect is associated with the relative position of the microcracks to the main crack tip. The minimum value of the $M$ integral was obtained for the model with two added microcracks. In particular, when the centre line of microcracks is ahead of the main crack, both shielding and amplification effects can be obtained as shown in Fig. 18. It is because the value of $M$-integral consists of two main contributing factors for three models. One is the propagation of the main crack and the microcracks, and the other is the interaction between the main crack and the microcracks. For the model shown in Fig. 17c, the position of microcracks is further away from the main crack surface/plane, which weakens the interaction between the microcracks and the main crack. Also, the damage induced by the propagation of these microcracks would increase the values of $M$-integral. For this situation, the shielding effect can only be observed for the model with two added microcracks, as indicated by the black line with blue symbols in Fig. 18. Also, irrespective of the positions of microcracks, the damage represented by the $M$-integral always increases with the increasing number of microcracks, due to the collective propagation of microcracks. This indicates a dependency of the shielding effect on the number of microcracks.

In addition, two different sizes of microcrack, i.e., $a=0.3 \mathrm{~mm}$ and $0.6 \mathrm{~mm}$, were considered to study the effect of microcrack length. The centreline of the microcracks was assumed to be vertically in line with the main crack tip. The number of microcracks also varied, between 2 and 32. As shown in Fig. 19, longer microcracks induce higher toughening effect in materials. Growth of the main crack is also presented for the model with two microcracks $(a=0.6 \mathrm{~mm})$, as shown in Fig. 20a. It can be seen that the main crack has a propagation of $2.7 \mathrm{~mm}$ at the 
left tip and $0.5 \mathrm{~mm}$ at the right tip at the end of loading step. For comparison, crack growth for the model with a single main crack is also shown in Fig. 20b, which clearly indicates that the growth of the main crack is weakened due to the growth of microcracks.

\section{Conclusions}

In this paper, the $M$-integral of a viscoplastic material containing single and multiple cracks was investigated using the extended finite element method. The results showed that the $M$ integral can be used as an effective parameter to evaluate the damage evolution during crack growth. Major conclusions are as follows:

(1) The $M$-integral is influenced by the viscoplastic material properties. In detail, for the same amount of crack growth, higher $M$-integral was obtained for a viscoplastic model compared to the pure elastic model due to the plastic deformation. Under creep loading condition, the results show that the increase of the $M$-integral is a slow and steady process. And for the loading rate investigation, we find that higher loading rate generally leads to increased values of the $M$-integral.

(2) For the model containing two cracks, the $M$-integral is affected by crack interaction, and strongly dependent on the relative positions and orientations of those interacting cracks. It can be concluded that collinear cracks produce the highest $M$-integral.

(3) The $M$-integral can be used to evaluate microcrack-toughening in viscoplastic materials. The results showed that the relative position of microcracks to the main crack tip would significantly affect the value of the $M$-integral. Maximum shielding effect was observed for two-microcrack model, especially when the centre line of microcracks were located behind the main crack tip. When the microcracks are ahead of the main crack tip, both shielding and amplification effects were observed due to the varied number of microcracks. In addition, considering the effect of microcrack length, longer microcracks induced higher toughening effect in materials. 


\section{Acknowledgments}

This work was supported by the National Natural Science Foundation of China (Nos. 11572235, 11772245, 11472205), Natural Science Basic Research Plan in Shaanxi Province of China (Program No. 2014K10-16), and the Fundamental Research Funds for the central Universities in China.

\section{References}

[1] Bouhala L, Shao Q, Koutsawa Y, et al. An XFEM crack-tip enrichment for a crack terminating at a bimaterial interface. Eng Fract Mech 2013; 102: 218-51-64.

[2] Chiang CR. Stress concentration of a crack-like spheroidal cavity lying on the prism plane of hexagonal crystal. Eng Fract Mech 2017; 184: 218-226.

[3] Dewapriya MAN, Meguid SA, Rajapakse RKND. Atomistic modelling of crack-inclusion interaction in grapheme. Eng Fract Mech 2018; 195: 92-103.

[4] Sutula D, Kerfriden P, Dam T, et al. Minimum energy multiple crack propagation. Part II: Discrete solution with XFEM. Eng Fract Mech 2018; 191: 225-256.

[5] Sutula D, Kerfriden P, Dam T, et al. Minimum energy multiple crack propagation. Part III: XFEM computer implementation and applications. Eng Fract Mech 2018; 191: 225-256.

[6] Guo LC, Kitamura T, Yan YB, et al. Fracture mechanics investigation on crack propagation in the nano-multilayered materials. Int J Solids Struc 2015; 64-65: 208-220.

[7] Lee CS, Yoo BM, Kim MH, Lee JM. Viscoplastic damage model for austenitic stainless steel and its application to the crack propagation problem at cryogenic temperatures. Int J Damage Mech 2013; 22(1): 95-115.

[8] Chen YT, Liu KX. Crack propagation in viscoplastic polymers: heat generation in near-tip zone and viscoplastic cohesive model. Appl Phys Lett 2015; 106(6): 061908.

[9] Chaboche JL. Constitutive equations for cyclic plasticity and cyclic viscoplasticity. Int J Plasticity $1989 ; 5(3): 247-302$

[10]Zhao LG, Tong J, Hardy MC. Prediction of crack growth in a nickel-based superalloy under fatigueoxidation conditions. Eng Fract Mech 2010; 77(6): 925-938. 
[11]Farukh F, Zhao LG, Jiang R, Reed P, Proprentner D, Shollock B. Fatigue crack growth in a nickelbased superalloy at elevated temperature-experimental studies, viscoplasticity modelling and XFEM predictions. Mech Adv Mater Modern Processes 2015; 1(1): 2.

[12]Hong YS, Liu XL, Lei ZQ, et al. The formulation mechanism of characteristic region at crack initiation for very-high-cycle fatigue of high-strength. Int J Fatig 2016; 89: 108-118.

[13]Qian GA, Lei WS, Peng L, et al. Statistical assessment of notch toughness against cleavage fracture of ferritic steels. Fatig Fract Eng Mater Struc 2017; 41: 1120-1131.

[14]Hong YS, Sun CQ. The nature and the mechanism of crack initiation and early growth for very-highcycle fatigue of metallic materials - and overview. Theor Appl Fract Mech 2017; 92: 331-350.

[15]Qian GA, Gao Y, Niffenegger M, et al. Comparison of constraint analyses with global and local approaches under uniaxial and biaxial loading. Euro J Mech/ A Solids 2018; 69: 135-146.

[16]Qian GA, Lei WS, Niffenegger M, et al. On the temperature independence of statistical model parameters for cleavage fracture in ferritic steels. Philos Mag 2018; 98(11): 959-1004.

[17]Dolbow JE, Gosz M. On the computation of mixed-mode stress intensity factors in functionally graded materials. Int J Solid Struct 2002; 39: 2557-2574.

[18]Yu HJ, Wu LZ, Guo LC, Du SY, He QL. Investigation of mixed-mode stress intensity factors for nonhomogeneous materials using an interaction integral method. Int J Solid Struct 2009; 46: 37103724.

[19]Yu HJ, Kitamura T. A new domain-independent interaction integral for solving the stress intensity factors of the materials with complex thermo-mechanical interfaces. Euro J Mech A-Solid 2015; 49: 500-509.

[20]Rice JR, Budiansky B. Conservation laws and energy-release rates. J Appl Mech 1973; 40: 201-203.

[21]Seed GM. The Boussinesq wedge and the $J_{k}, L$ and $M$ integrals. Fatigue Fract Eng Mater Struct 1997; 20(6): 907-916.

[22]Chen YH. M-integral analysis for two-dimensional solids with strongly interacting microcracks. Part I: in an infinite brittle solid. Int J Solids Struct 2001; 38(18): 3193-3212.

[23]Chang JH, Lin JS. Surface energy for creation of multiple curved cracks in rubbery materials. J Appl Mech 2007; 74(3): 488-496. 
[24]Yu NY, Li Q. Failure theory via the concept of material configurational forces associated with the $M$ integral. Int J Solids Struct 2013; 50(25): 4320-4332.

[25]Chang JH, Kang YC, Chung LG. M- and Mc-integrals for multicracked problems in three dimensions. J Eng Mech 2013; 139(12): 1874-1880.

[26]Yang Q, Cox B. Cohesive models for damage evolution in laminated composites. Int J Fract 2005; 133(2): 107-137.

[27]Stolarska M, Chopp DL, Moës N, Belytschko T. Modelling crack growth by level sets in the extended finite element method. Int J Numer Meth Eng 2001; 51(8): 943-960.

[28]Bellec J, Dolbow JE. A note on enrichment functions for modelling crack nucleation. Commun Numer Meth Eng 2003; 19(12): 921-932.

[29]Barbieri E, Petrinic N, Meo M, Tagarielli VL. A new weight-function enrichment in meshless methods for multiple cracks in linear elasticity. Int J Numer Meth Eng 2012; 90(2): 177-195.

[30]Daux C, Moes N, Dolbow J, Sukumar N, Belytschko T. Arbitrary branched and intersecting cracks with the extended finite element method. Int J Numer Meth Eng 2000; 48(12): 1741-1760.

[31]Budyn E, Zi G, Moës N, Belytschko T. A method for multiple crack growth in brittle materials without remeshing. Int J Numer Meth Eng 2004; 61(10): 1741-1770.

[32] Singh IV, Mishra BK, Bhattacharya S, Patil RU. The numerical simulation of fatigue crack growth using extended finite element method. Int J Fatig 2012; 36(1): 109-119.

[33]Xu D, Liu Z, Liu X, Zeng Q, Zhuang Z. Modelling of dynamic crack branching by enhanced extended finite element method. Comput Mech 2014; 54(2): 489-502.

[34]Kästner M, Haasemann G, Ulbricht V. Multiscale modelling of the effective viscoplastic material behaviour of textile-reinforced polymers using XFEM. Int J Numer Meth Eng 2010; 10(1): 297-298.

[35]Liu X, Waisman H, Fish J. A new crack tip enrichment function in the extended finite element method for general inelastic materials. Int J Multiscale Com 2012; 10: 343-360.

[36]Bergara A, Doradob JI, Martin-Meizosoa A, Martínez-Esnaola JM. Fatigue crack propagation in complex stress fields: Experiments and numerical simulations using the Extended Finite Element Method (XFEM). Int J Fatig 2017; 103: 112-121.

[37]Eshelby JD. The elastic energy-momentum tensor. J Elasticity 1975; 5(3-4): 321-335. 
[38]Li Q, Hu YF, Chen YH. On the physical interpretation of the M-integral in nonlinear elastic defect mechanics. Int J Damage Mech 2013; 22(4): 602-613.

[39]Chen YH. M-integral analysis for two-dimensional solids with strongly interacting microcracks. Part I: in an infinite brittle solid. Int J Solids Struct 2001; 38(18): 3193-3212.

[40]Li Q, Guo YL, Hou JL, Zhu WJ. The M-integral based failure description on elasto-plastic materials with defects under biaxial loading. Mech Mater 2017; 112: 163-171.

[41]Moran B, Shih CF. Crack tip and associated domain integrals from momentum and energy balance. Eng Fract Mech 1987; 27(6): 615-642.

[42]Nikishkov GP, Atluri SN. An equivalent domain integral method for computing crack-tip integral parameters in non-elastic, thermo-mechanical fracture. Eng Fract Mech 1987; 26(6): 851-867.

[43] Shivakumar KN, Raju IS. An equivalent domain integral method for three-dimensional mixed-mode fracture problems. Eng Fract Mech 1992; 42(6): 935-959.

[44] Zhang X, Mai YW, Jeffrey RG. A cohesive plastic and damage zone model for dynamic crack growth in rate-dependent materials. Int J Solids Struct 2003; 40(21): 5819-5837.

[45] Liu X. A novel discrete damage zone model and enhancement of the extended finite element method for fracture mechanics problems. Dissertations \& Thesis - Gradworks, 2012.

[46]Moes N, Dolbow J, Belytschko T. A finite element method for crack growth without remeshing. Int J Numer Meth Eng 1999; 46(1): 131-150.

[47]Kästner M, Haasemann G, Ulbricht V. Multiscale XFEM-modelling and simulation of the inelastic material behavior of textile-reinforced polymers. Int J Numer Meth Eng 2011; 86(4-5): 477-498.

[48]Kästner M, Obst M, Brummund J, et al. Multiscale Modelling of the effective viscoplastic material behaviour of textile-reinforced polymers using XFEM. Pamm 2015; 10(1): 297298. 
[49]Singh R, Mahajan D K. Crystal Orientation Effect on SIF in Single Crystals: A Study Based on Coupled Framework of XFEM and Crystal Plasticity Model[M]// Proceedings of Fatigue, Durability and Fracture Mechanics. 2018.

[50]Jiang R, Karpasitis N, Gao N, Reed PAS. Effects of microstructures on fatigue crack initiation and short crack propagation at room temperature in an advanced disc superalloy. Mater Sci Eng A 2015; 641: 148-159.

[51]Jiang R, Gao N, Reed PAS. Influence of orientation-dependent grain boundary oxidation on fatigue cracking behaviour in an advanced Ni-based superalloy. J Mater Sci 2015; 50(12): 4379-4386.

[52]Jiang R, Bull DJ, Proprentner D, Shollock B, Reed PAS. Effects of oxygen-related damage on dwellfatigue crack propagation in a P/M Ni-based superalloy: from 2D to 3D assessment. Int J Fatig 2017; 99: 175-186.

[53]Hutchinson JW. Crack tip shielding by micro-cracking in brittle solids. Acta Metall 1987; 35(7): 16051619.

[54] Chow CL, Lu TJ. A continuum damage mechanics approach to crack tip shielding in brittle solids. Int J Fract 1991; 50(2): 79-114.

[55] Loehnert S, Belytschko T. A multiscale projection method for macro/microcrack simulations. Int J Numer Meth Eng 2007; 71:1466-1482.

[56] Guidault PA, Allix O, Champaney L, Cornuault C. A multiscale extended finite element method for crack propagation. Comput Meth Appl Mech Eng 2008; 197(5):381-399.

[57] Svenning E, Fagerström M, Larsson F. Modeling microcracks using weak periodic boundary conditions and XFEM. Proceedings of NSCM-27: the 27th Nordic Seminar on Computational Mechanics. 2014. 


\section{List of Table Captions}

Table 1 Optimised parameter values for the viscoplastic constitutive model [12].

Table 2 Values of the $M$-integral for ten different paths at the end of loading (time $100 \mathrm{~s}$ ).

Table 3 Values of the $M$-integral for different orientation of Crack 2. 
Table 1 Optimised parameter values for the viscoplastic constitutive model [12].

\begin{tabular}{cc}
\hline Parameters & Optimised values \\
\hline$E(\mathrm{GPa})$ & 178.773 \\
$k(\mathrm{MPa})$ & 126.23 \\
$b$ & 6.37 \\
$Q(\mathrm{MPa})$ & 171.49 \\
$a_{1}(\mathrm{MPa})$ & 272.45 \\
$C_{1}$ & 2123.61 \\
$a_{2}(\mathrm{MPa})$ & 306.78 \\
$C_{2}$ & 2587.69 \\
$Z$ & 2018.32 \\
$n$ & 5.17 \\
\hline
\end{tabular}


Table 2 Values of the $M$-integral for ten different paths at the end of loading (time $100 s$ ).

\begin{tabular}{lccccc}
\hline Path & 1 & 2 & 3 & 4 & 5 \\
\hline Dimension & $\begin{array}{c}\text { length }=25 \mathrm{~mm} \\
\text { width }=26.5 \mathrm{~mm}\end{array}$ & $\begin{array}{c}\text { length }=25.2 \mathrm{~mm} \\
\text { width }=26.3 \mathrm{~mm}\end{array}$ & $\begin{array}{c}\text { length }=25.4 \mathrm{~mm} \\
\text { width }=26.1 \mathrm{~mm}\end{array}$ & $\begin{array}{c}\text { length }=25.6 \mathrm{~mm} \\
\text { width }=26 \mathrm{~mm}\end{array}$ & $\begin{array}{c}\text { length }=25.8 \mathrm{~mm} \\
\text { width }=25.8 \mathrm{~mm}\end{array}$ \\
\hline $\begin{array}{c}M \text {-integral } \\
(\mathrm{J} / \mathrm{m})\end{array}$ & 27.24 & 27.31 & 27.43 & 27.52 & 27.23 \\
\hline \hline Path & 6 & 7 & 8 & 9 & 10 \\
\hline Dimension & $\begin{array}{c}\text { length }=26 \mathrm{~mm} \\
\text { width }=26 \mathrm{~mm}\end{array}$ & $\begin{array}{c}\text { length }=26.2 \mathrm{~mm} \\
\text { width }=25.6 \mathrm{~mm}\end{array}$ & $\begin{array}{c}\text { length }=26.4 \mathrm{~mm} \\
\text { width }=25.4 \mathrm{~mm}\end{array}$ & $\begin{array}{c}\text { length }=26.5 \mathrm{~mm} \\
\text { width }=25.2 \mathrm{~mm}\end{array}$ & $\begin{array}{c}\text { length }=25 \mathrm{~mm} \\
\text { width }=25 \mathrm{~mm}\end{array}$ \\
\hline $\begin{array}{c}M \text {-integral } \\
(\mathrm{J} / \mathrm{m})\end{array}$ & 27.30 & 27.43 & 27.53 & 27.30 & 27.37 \\
\hline
\end{tabular}


Table 3 Values of the $M$-integral for different orientation of Crack 2.

\begin{tabular}{cccccccc}
\hline$\alpha$ & $0^{\circ}$ & $30^{\circ}$ & $45^{\circ}$ & $60^{\circ}$ & $90^{\circ}$ & $120^{\circ}$ & $150^{\circ}$ \\
\hline$M$-integral & & & & & & & \\
$(\mathrm{J} / \mathrm{m})$ & 115.54 & 39.09 & 51.28 & 38.37 & 27.05 & 38.30 & 38.96 \\
\hline
\end{tabular}




\section{List of Figure Captions}

Fig. 1. Multiple cracks with integral contour in a 2D plane.

Fig. 2. Sketch of domain integral method for the $M$-integral calculation.

Fig. 3. A viscoplastic plate with randomly distributed multi-cracks in a local damage zone.

Fig. 4. Sketch of nodal enrichment and crack tip polar coordinate system

Fig. 5. Simulated strain-rate effects of four strain rates $\left(0.001 / \mathrm{s}, 0.01 / \mathrm{s}, 0.1 / \mathrm{s}\right.$ and $\left.8.3 \times 10^{-5} / \mathrm{s}\right)$ for monotonic tensile (maximum strain 1\%), and a comparison of the simulated response and experimental data for a strain rate of $8.3 \times 10^{-5} / \mathrm{s}$.

Fig. 6. (a) A sketch of the geometrical model and (b) finite element mesh.

Fig. 7. Evolution of the $M$-integral for elastic and viscoplastic material models.

Fig. 8. The $M$-integral versus the crack growth length for two material models.

Fig. 9. Loading condition and the corresponding variation of $M$-integral

Fig. 10. The distributions of von-Mises stress for different times

Fig. 11. The distributions of displacement for different times

Fig. 12. The variation of $M$-integral for three different loading rates.

Fig. 13. The variation of $M$-integral against crack growth for three different loading rates.

Fig. 14. Sketch of damage zone with two interacting cracks

Fig. 15. Evolution of the $M$-integral for different orientations of Crack 2.

Fig. 16. Growth profile for two collinear cracks at the end of loading step (a deformation scale factor of 10).

Fig. 17. The model containing a vertical row of microcracks with the centres (a) behind, (b) in line with and (c) ahead of the right tip of the main crack.

Fig. 18. The $M$-integral for different numbers of microcracks with centres behind, in line with, and ahead of the main crack tip. 
Fig. 19. The $M$-integral for different numbers of microcracks with a length of $0.3 \mathrm{~mm}$ and 0.6 $m m$, respectively.

Fig. 20. Crack propagation captured by XFEM at the end of loading step: (a) a main crack with two microcracks (b) a single main crack and (a deformation scale factor of 10). 


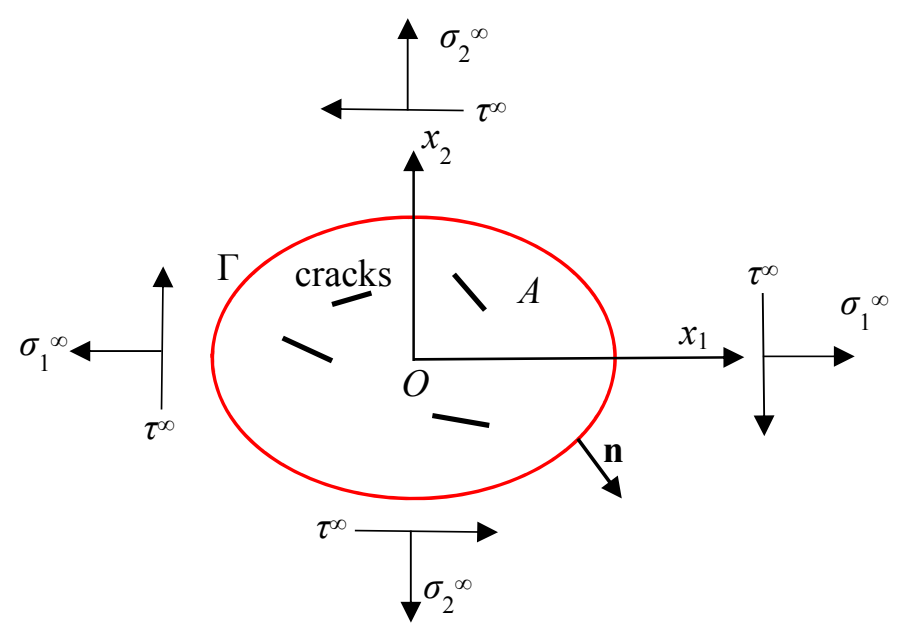

Fig. 1. Multiple cracks with integral contour in a 2D plane. 


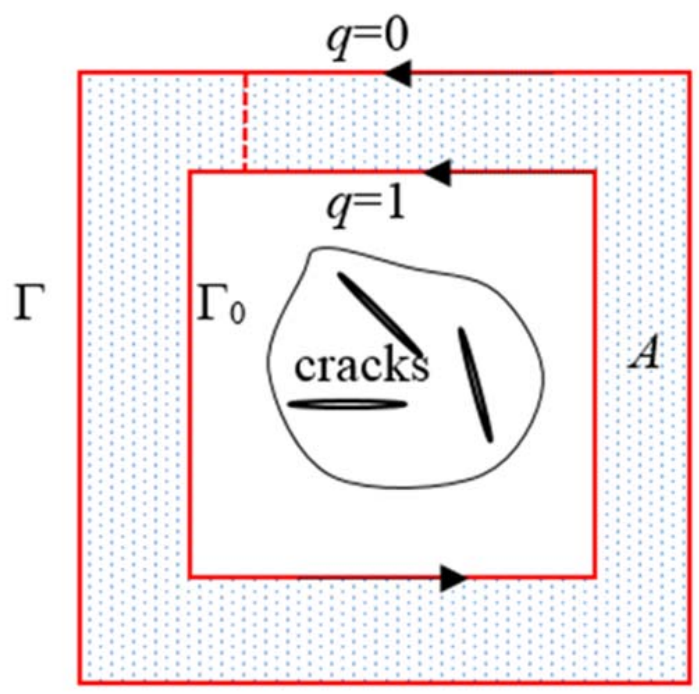

Fig. 2. Sketch of domain integral method for the $M$-integral calculation. 




Fig. 3. A viscoplastic plate with randomly distributed multi-cracks in a local damage zone. 


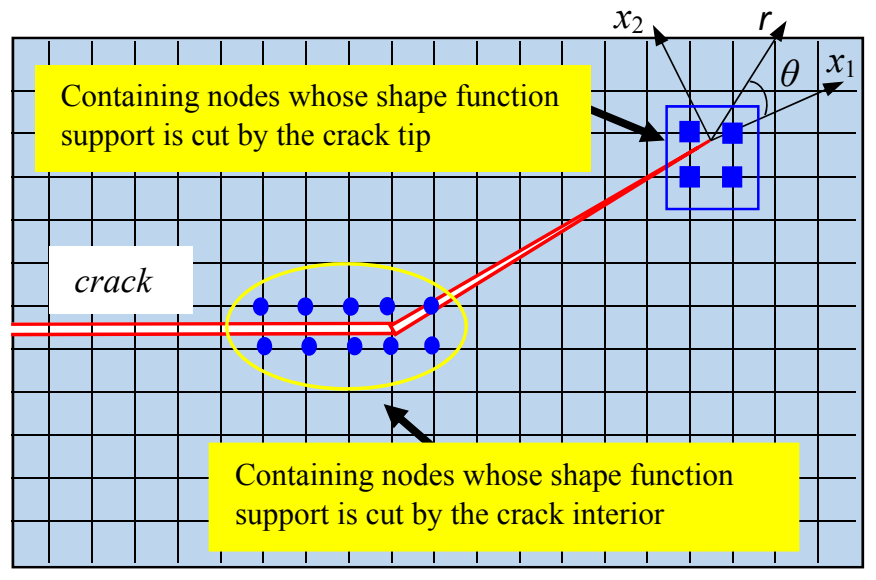

Fig. 4. Sketch of nodal enrichment and crack tip polar coordinate system 


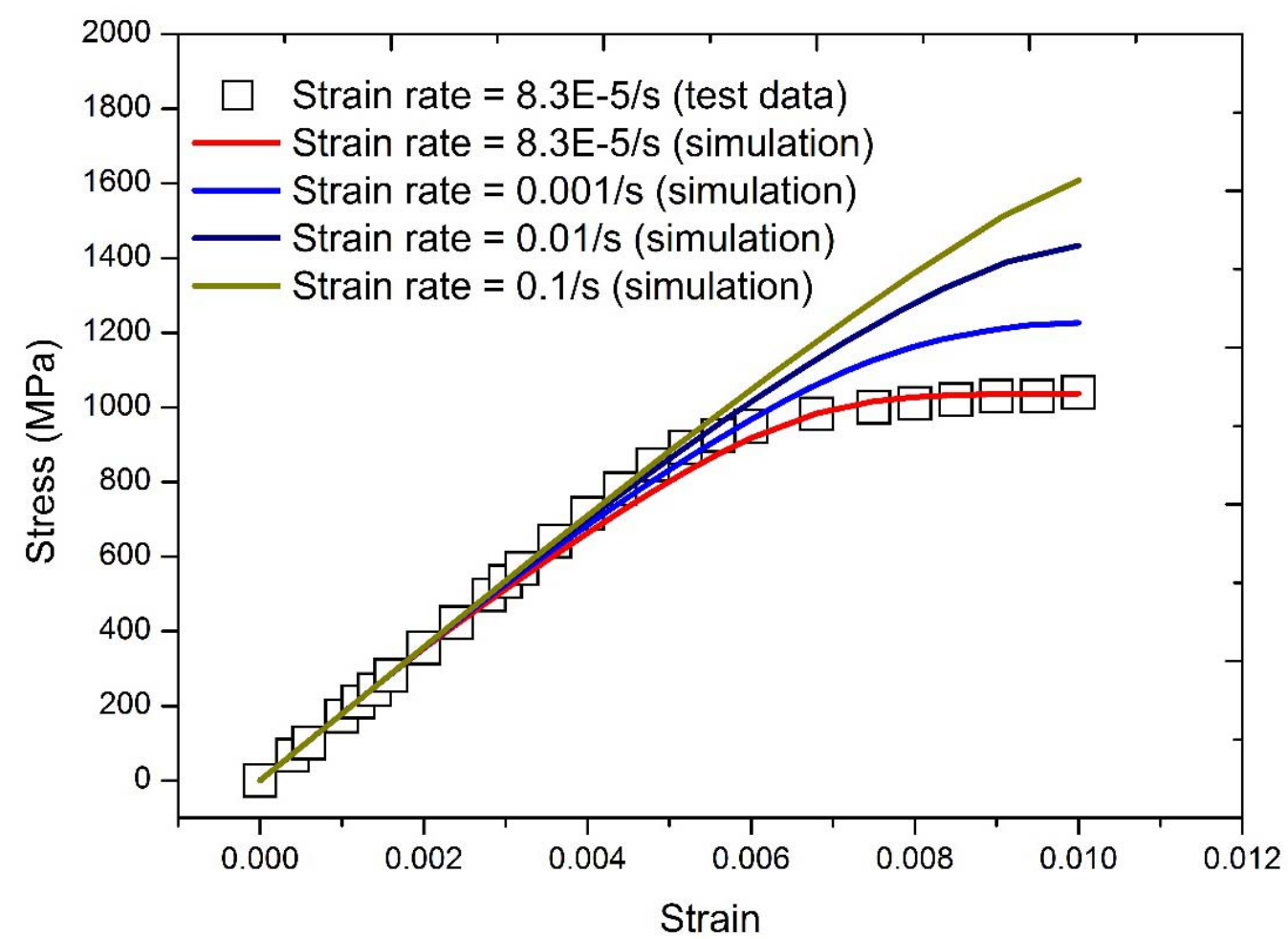

Fig. 5. Simulated strain-rate effects of four strain rates $\left(0.001 / \mathrm{s}, 0.01 / \mathrm{s}, 0.1 / \mathrm{s}\right.$ and $\left.8.3 \times 10^{-5} / \mathrm{s}\right)$ for monotonic tensile (maximum strain 1\%), and a comparison of the simulated response and experimental data for a strain rate of $8.3 \times 10^{-5} / \mathrm{s}$. 


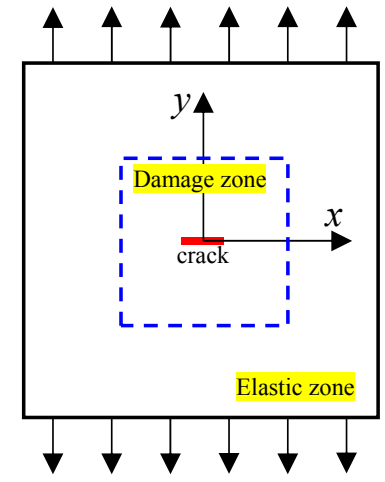

(a)

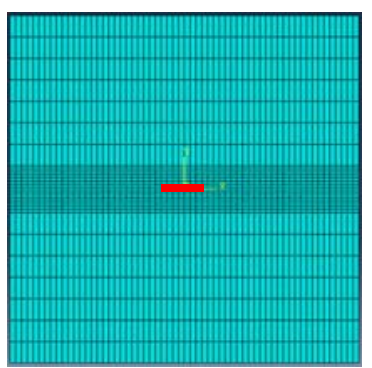

(b)

Fig. 6. (a) A sketch of the geometrical model and (b) finite element mesh. 


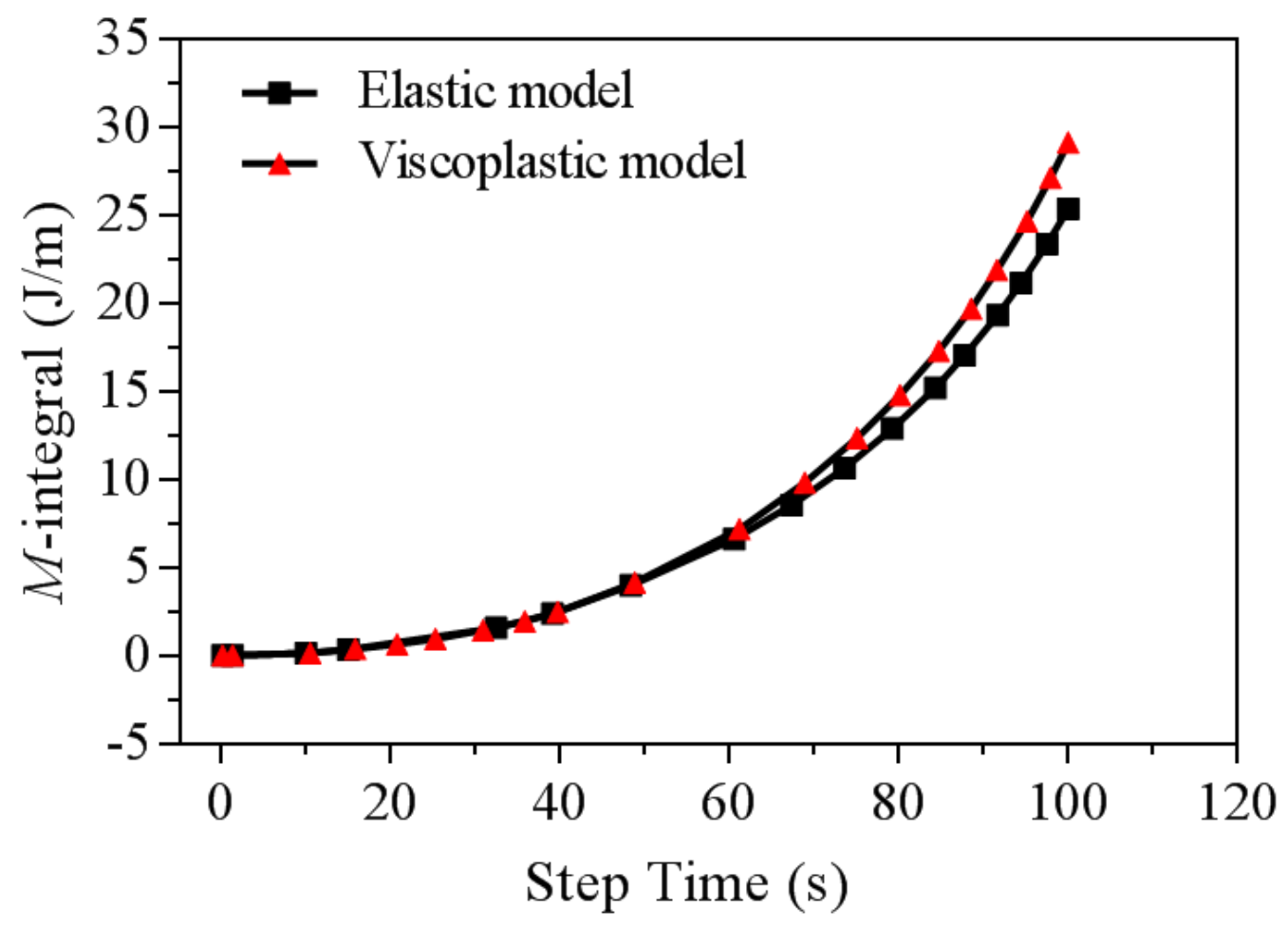

Fig. 7. Evolution of the $M$-integral for elastic and viscoplastic material models. 




Fig. 8. The $M$-integral versus the crack growth length for two material models. 


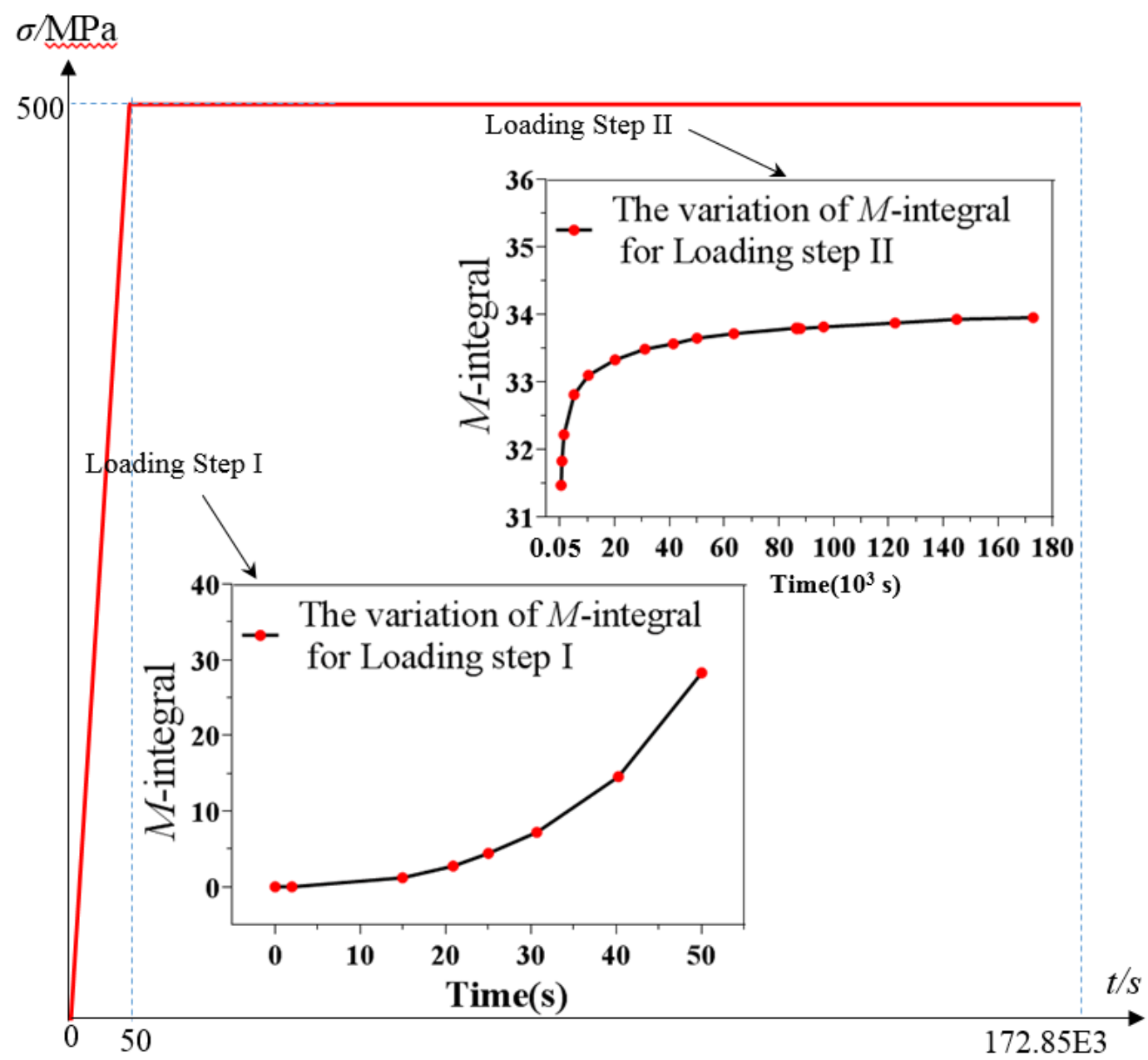

Fig. 9. Loading condition and the corresponding variation of $M$-integral 


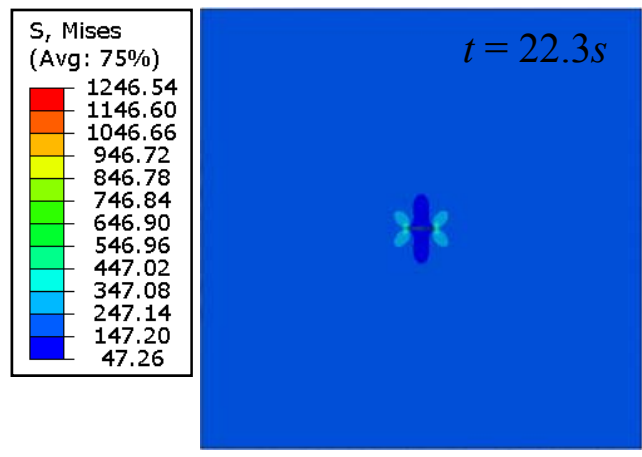

(a)

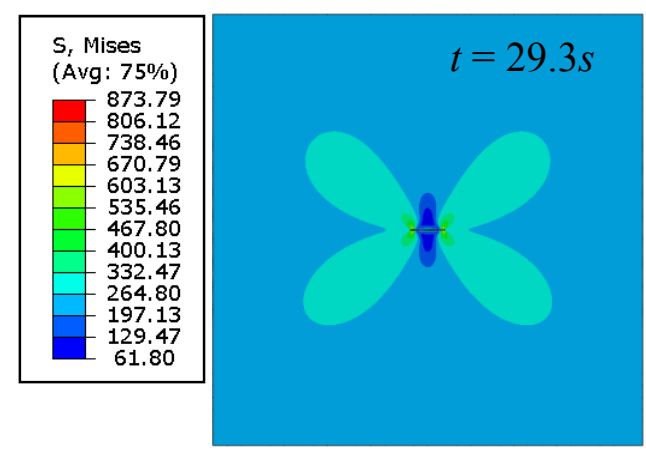

(c)

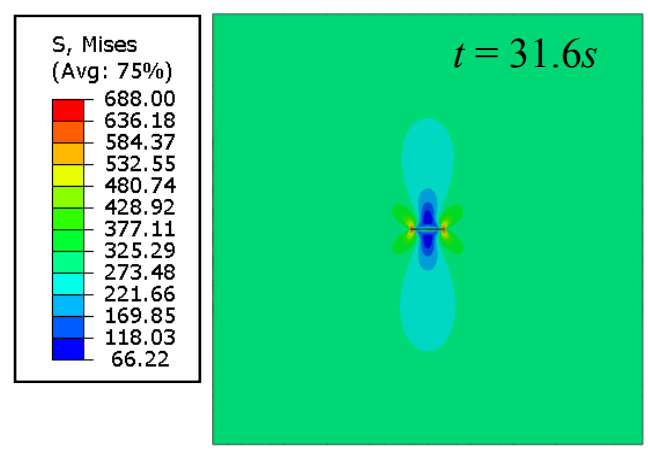

(e)



$(\mathrm{g})$

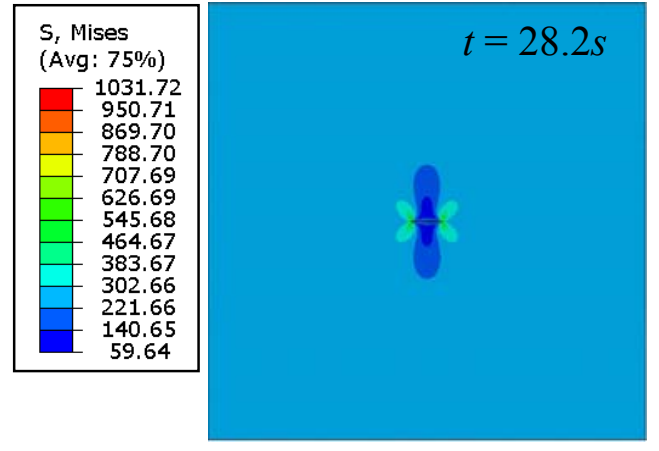

(b)

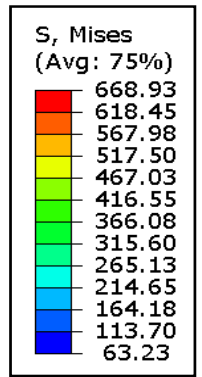

(d)

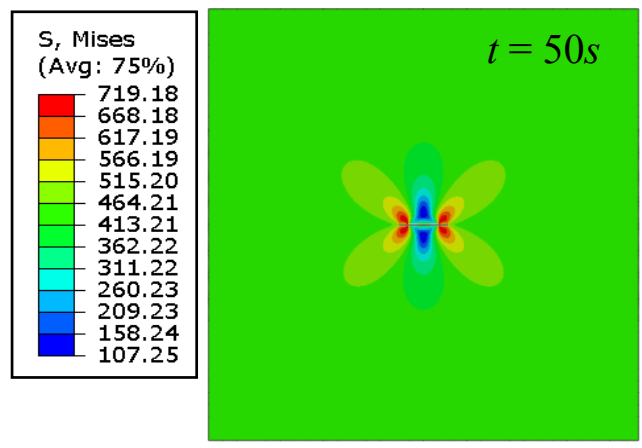

(f)

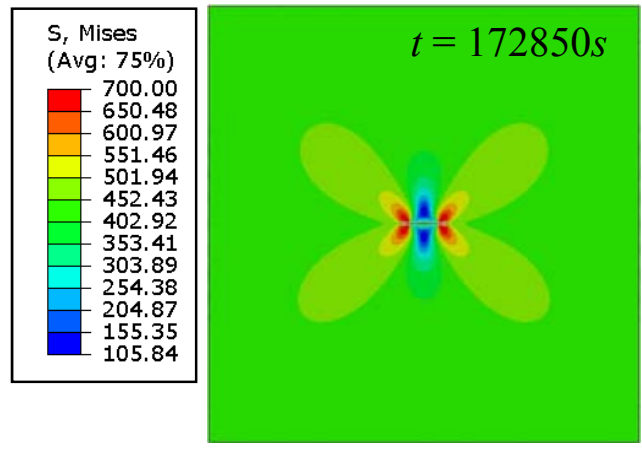

(h)

Fig. 10. The distributions of von-Mises stress for different times (a) $t=22.3 s$, (b) $t=28.2 s$, (c) $t=29.3 \mathrm{~s}$, (c) $t=30.1 \mathrm{~s}$, (e) $t=31.6 \mathrm{~s}$, (f) $t=50 \mathrm{~s}$, (g) $t=53 \mathrm{~s}$ and (h) $t=172850 \mathrm{~s}$. 


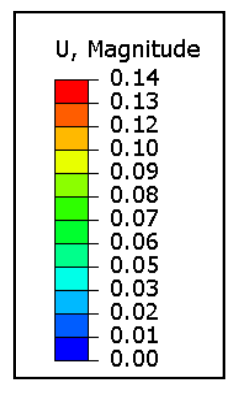

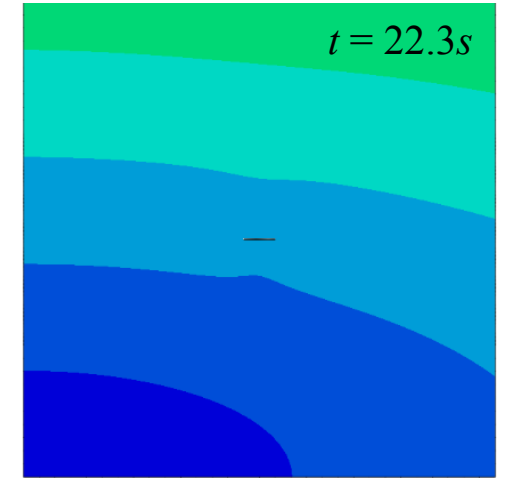

(a)



(c)

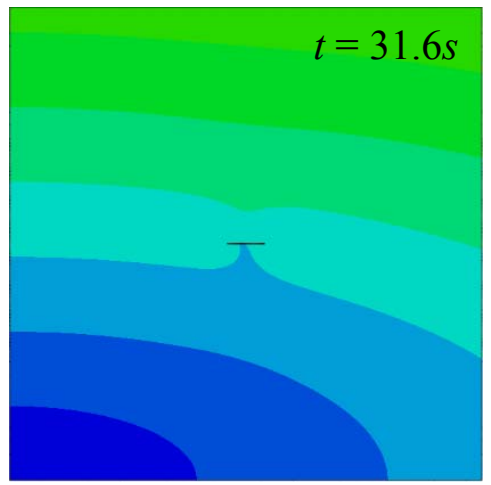

(e)

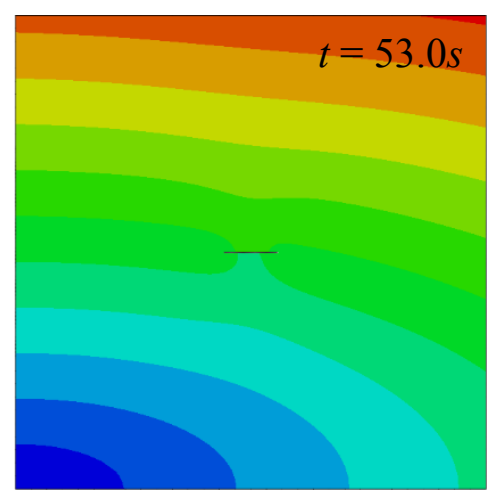

$(\mathrm{g})$

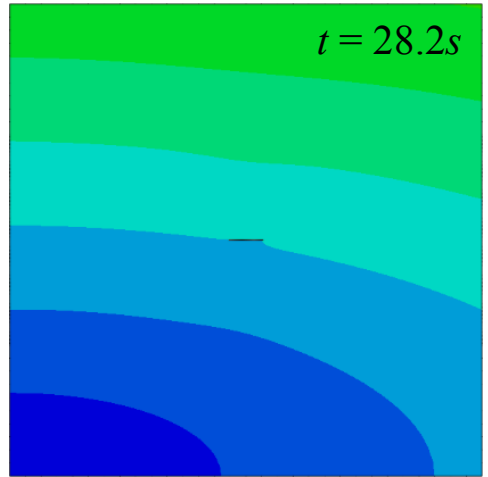

(b)

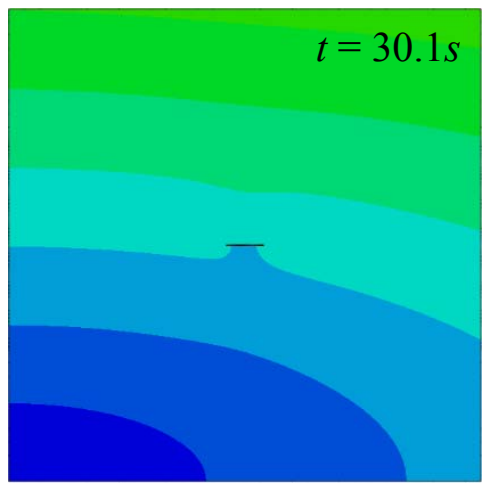

(d)

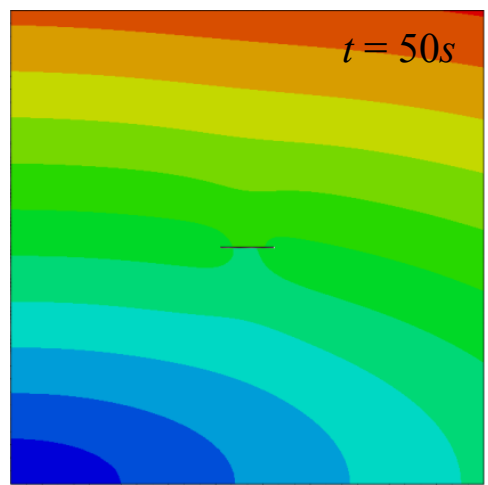

(f)

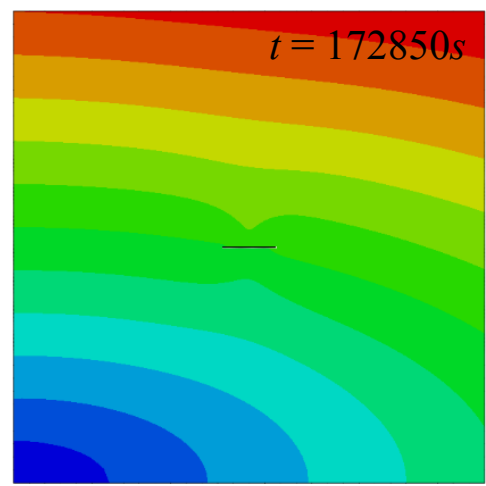

(h)

Fig. 11. The distributions of displacement for different times (a) $t=22.3 \mathrm{~s}$, (b) $t=28.2 \mathrm{~s}$, (c) $t=29.3 \mathrm{~s}$, (c) $t=30.1 \mathrm{~s}$, (e) $t=31.6 \mathrm{~s}$, (f) $t=50 \mathrm{~s}$, (g) $t=53 \mathrm{~s}$ and (h) $t=172850 \mathrm{~s}$. 


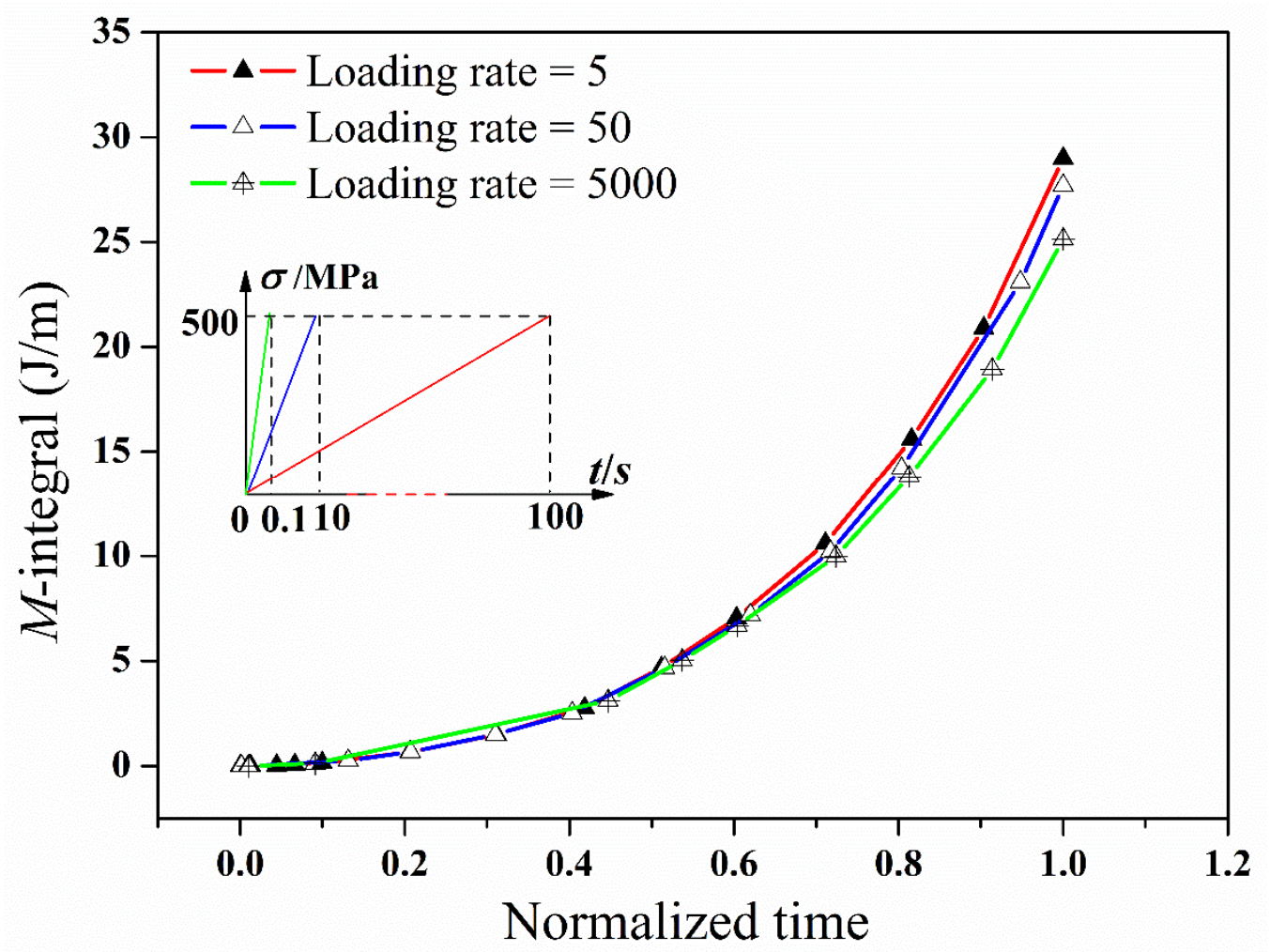

Fig. 12. The variation of $M$-integral for three different loading rates. 




Fig. 13. The variation of $M$-integral against crack growth for three different loading rates. 


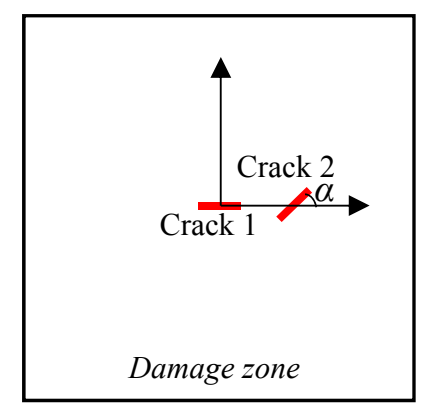

Fig. 14. Sketch of damage zone with two interacting cracks 


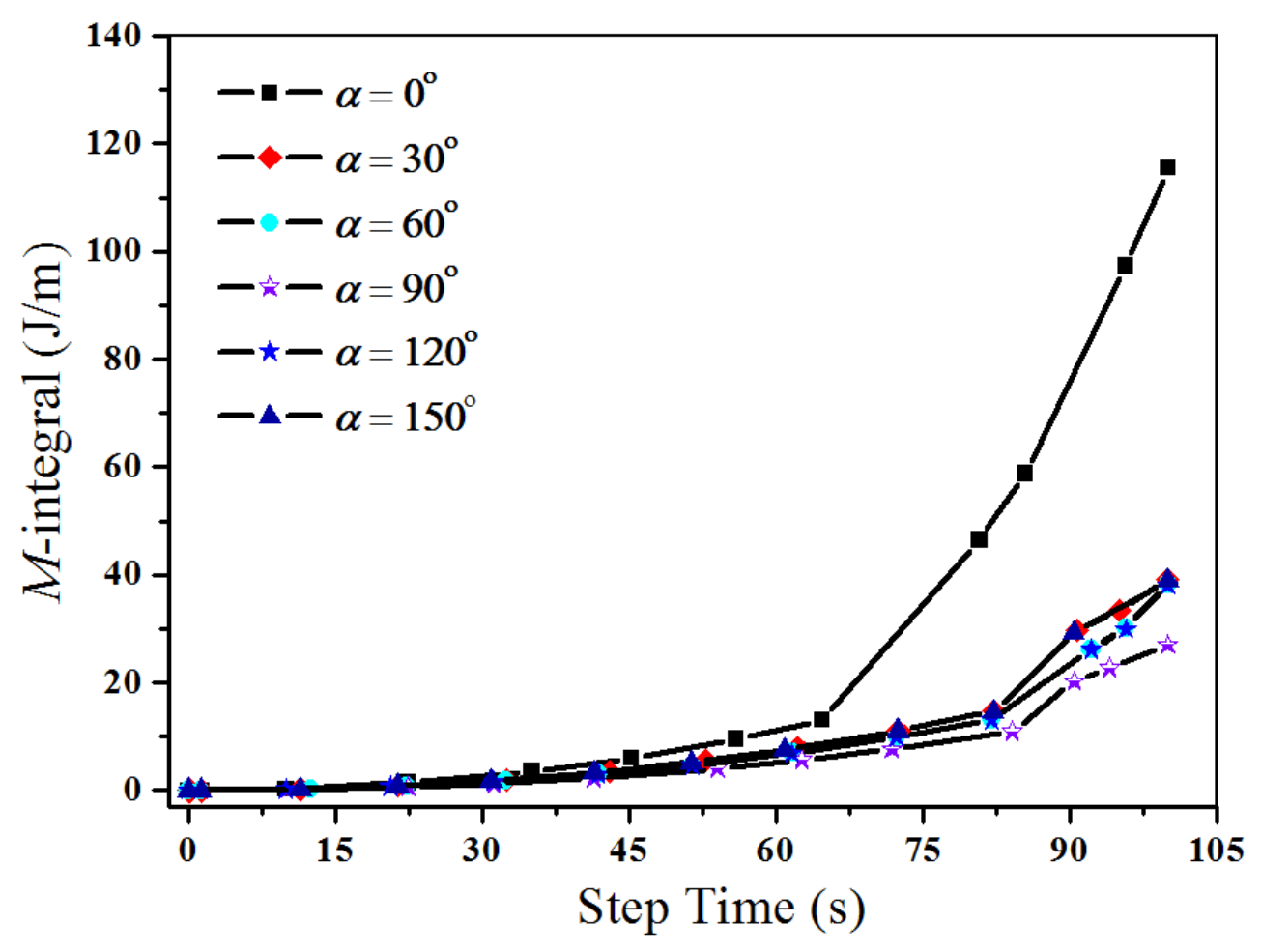

Fig. 15. Evolution of the $M$-integral for different orientations of Crack 2. 


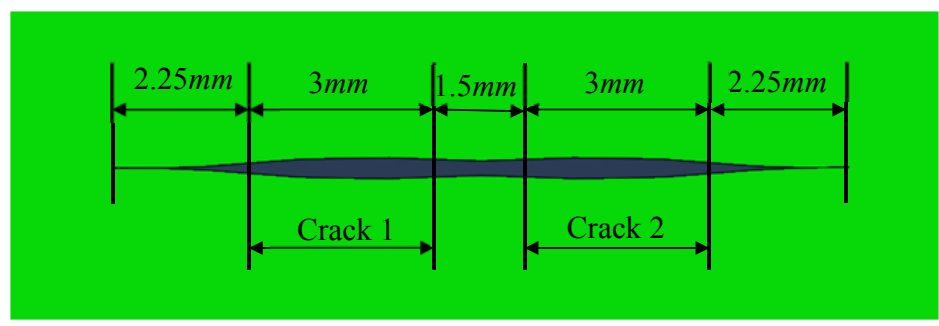

Fig. 16. Growth profile for two collinear cracks at the end of loading step

(a deformation scale factor of 10). 


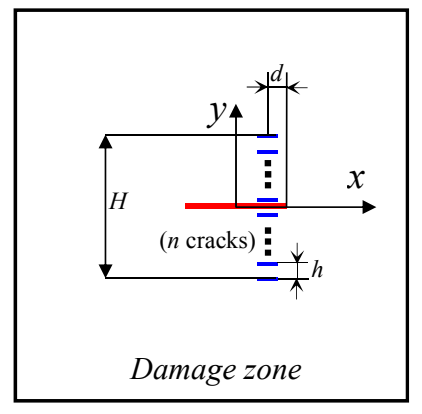

(a)

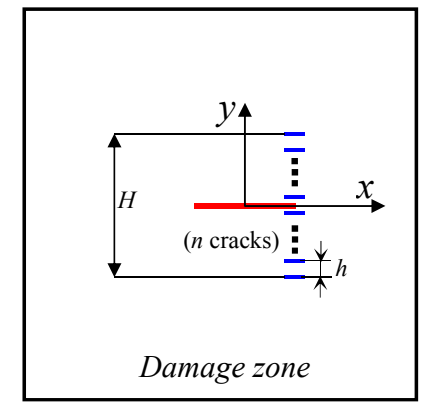

(b)

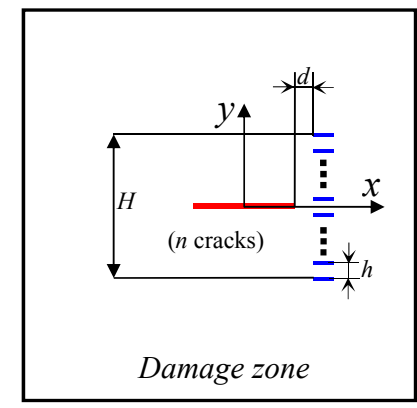

(c)

Fig. 17. The model containing a vertical row of microcracks with the centres (a) behind, (b) in line with and (c) ahead of the right tip of the main crack. 


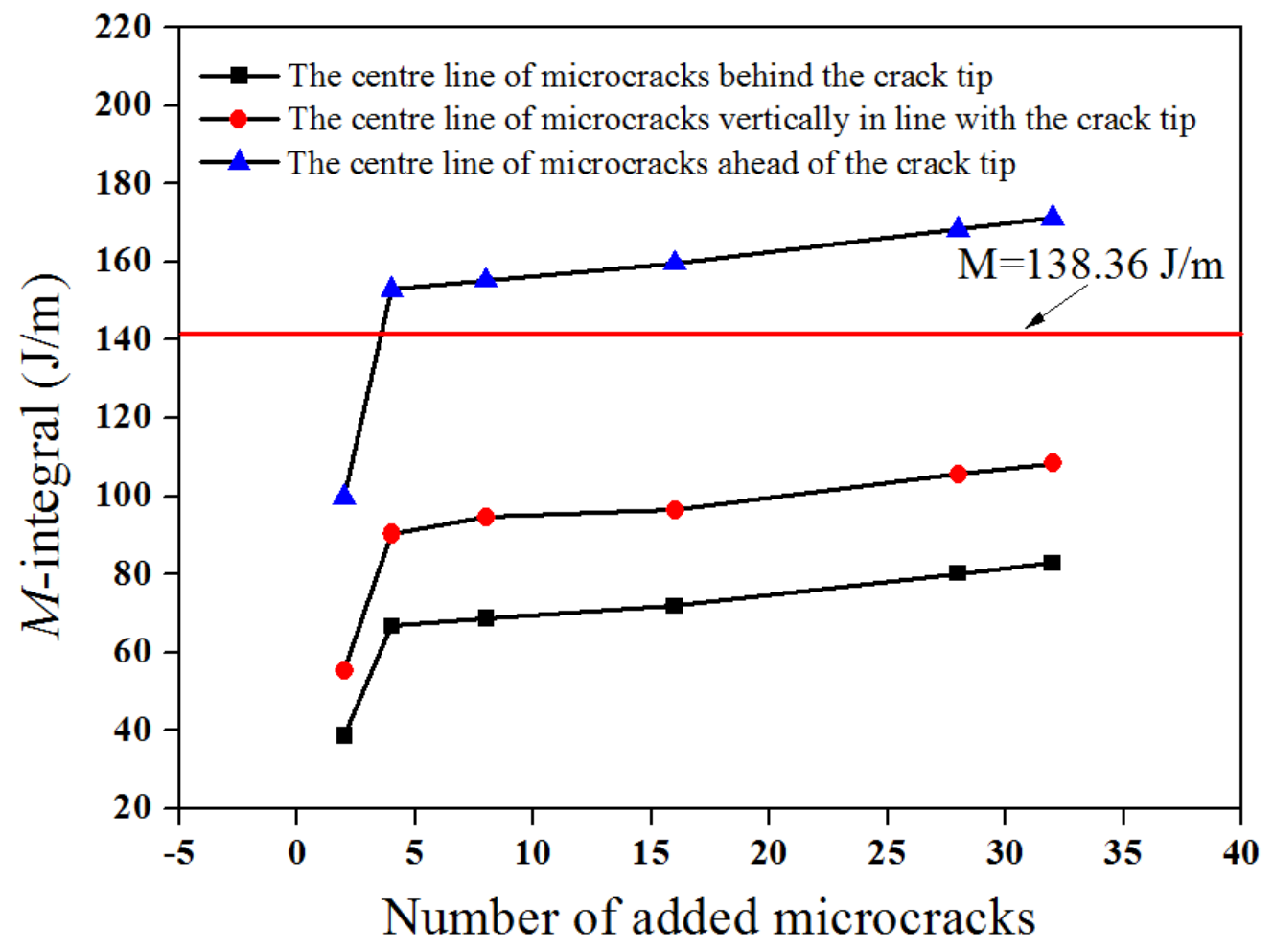

Fig. 18. The $M$-integral for different numbers of microcracks with centres behind, in line with, and ahead of the main crack tip. 


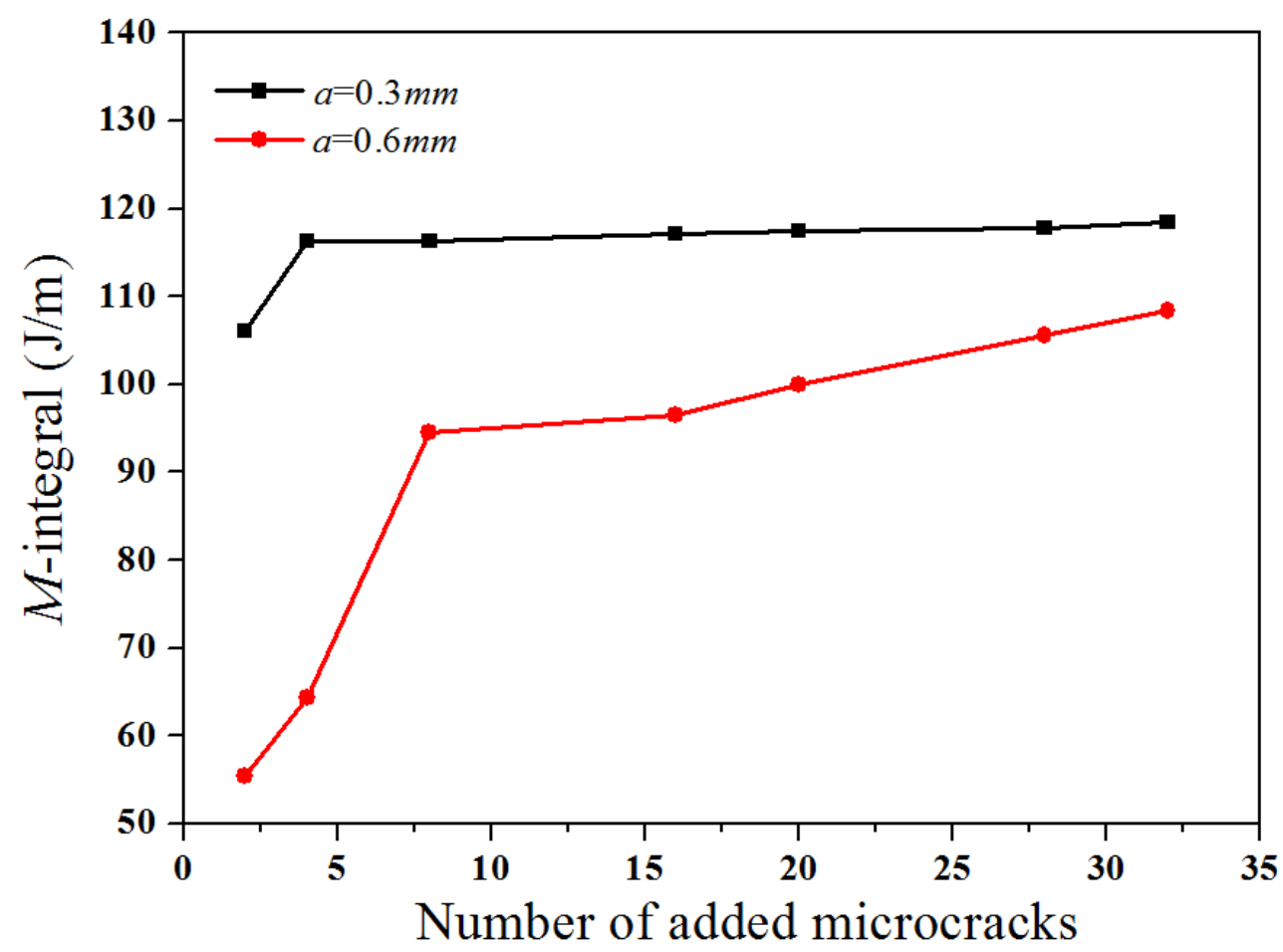

Fig. 19. The $M$-integral for different numbers of microcracks with a length of $0.3 \mathrm{~mm}$ and 0.6 $m m$, respectively. 


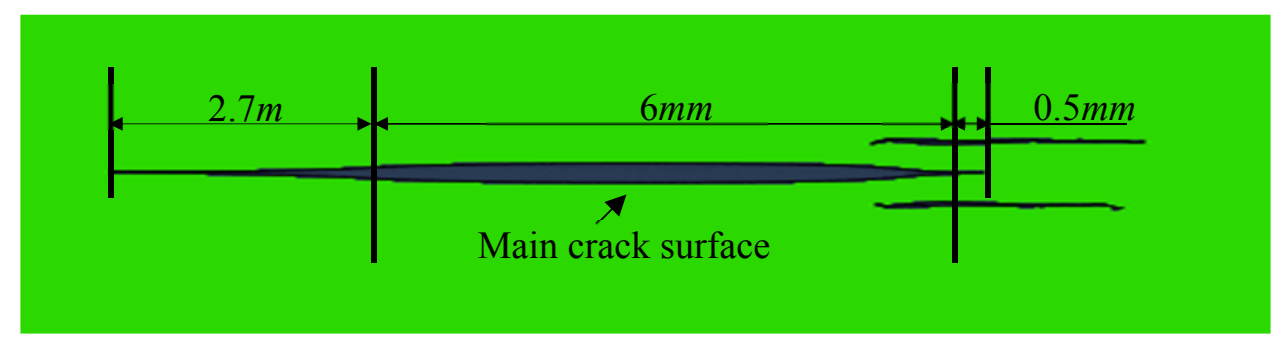

(a)

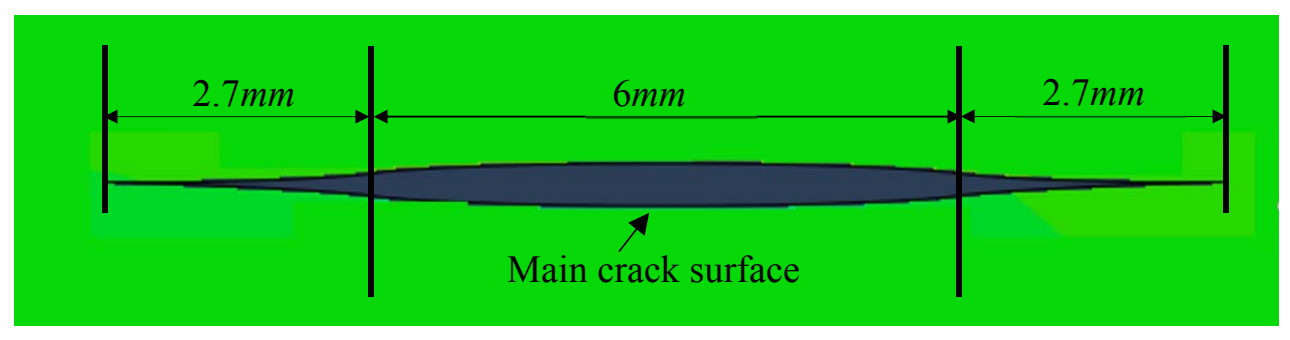

(b)

Fig. 20. Crack propagation captured by XFEM at the end of loading step: (a) a main crack with two microcracks (b) a single main crack and (a deformation scale factor of 10). 